\title{
A Novel Framework for Backstepping-Based Control of Discrete-Time Strict-Feedback Nonlinear Systems with Multiplicative Noises
}

\author{
Min Wang, Zidong Wang, Hongli Dong and Qing-Long Han
}

\begin{abstract}
This paper is concerned with the exponential meansquare stabilization problem for a class of discrete-time strictfeedback nonlinear systems subject to multiplicative noises. The state-dependent multiplicative noise is assumed to occur randomly based on a stochastic variable obeying the Gaussian white distribution. To tackle the difficulties caused by the multiplicative noise, a novel backstepping-based control framework is developed to design both the virtual control laws and the actual control law for the original nonlinear system, and such a framework is fundamentally different from the traditional $n$-step predictor strategy. The proposed design scheme provides an effective way in establishing the relationship between the system states and the controlled errors, by which a noise-intensity-dependant stability condition is derived to ensure that the closed-loop system is exponentially mean-square stable for exactly known systems. To further cope with nonlinear modeling uncertainties, the radial basis function neural network (NN) is employed as a function approximator. In virtue of the proposed backstepping-based control framework, the ideal controller is characterized as a function of all system states, which is independent of the virtual control laws. Therefore, only one $\mathrm{NN}$ is employed in the final step of the backstepping procedure and, subsequently, a novel adaptive neural controller (with modified weight updating laws) is presented to ensure that both the neural weight estimates and the system states are uniformly bounded in the mean-square sense under certain stability conditions. The control performance of the proposed scheme is illustrated through simulation results.
\end{abstract}

Index Terms-Nonlinear systems, discrete-time strict-feedback systems, backstepping-based control, adaptive control, neural networks, multiplicative noises

\section{INTRODUCTION}

As a class of nonlinear systems in the triangular form [15], [31], the strict-feedback nonlinear systems (SFNSs) have attracted a great deal of attention in the past two decades since SFNSs are capable of modeling many practical systems such as hypersonic flight vehicles [41], chemical reaction processes [23] and marine surface vessels [5]. It is well known

This work was supported in part by the National Natural Science Foundation of China under Grants 61773169, 61873058, 61873148, 61933007 and 61973129 , the Outstanding Youth Program of the Guangdong Natural Science Foundation under Grant 2019B151502058, the Fundamental Research for the Central Universities of China, the Royal Society of the UK, and the Alexander von Humboldt Foundation of Germany. (Corresponding author: Min Wang)

M. Wang is with the School of Automation Science and Engineering, South China University of Technology, Guangzhou 510641, China. (e-mail: auwangmin@scut.edu.cn).

Z. Wang is with the Department of Computer Science, Brunel University London, Uxbridge, Middlesex, UB8 3PH, United Kingdom. (e-mail: Zidong.Wang@brunel.ac.uk).

H. Dong is with the Institute of Complex Systems and Advanced Control, Northeast Petroleum University, Daqing 163318, China. (e-mail: shiningdhl@vip.126.com)

Q.-L. Han is with the School of Software and Electrical Engineering, Swinburne University of Technology, Melbourne, VIC 3122, Australia. (email: qhan@swin.edu.au that, as a breakthrough technique in nonlinear control theory, the backstepping procedure [19] has become an extremely powerful tool for solving control problems of SFNSs. By applying the backstepping procedure, a systematic control design framework has been constructed for continuous-time SFNSs and a large number of results have been reported in the literature, see e.g. [3], [8] and the references therein. For deterministic SFNSs, the adaptive control strategies have been developed to identify uncertain/unknown system parameters [3], [17], [27]. Also, by combining neural networks (NNs) and fuzzy logic systems, approximated-based adaptive control schemes have been developed in [22], [39], [48] to handle nonlinear uncertainties. These methods have been further extended to continuous-time SFNSs with different phenomena including, but are not limited to, partial immeasurable states [26], [33], various time-delays [12], [37], [45], and state/output constraints [1], [4], [16], [32]. To deal with the control problem of a more general class of nonlinear systems, a specific backstepping procedure has been elegantly developed in [28] for generalized triangular systems with periodic dynamics. Based on the converse input-to-state stability Lyapunov theorems, the result in [28] has been successfully extended to generalized triangular systems with deterministic disturbances in [6], [7].

For decades, stochastic control has proven to be an active area of the mainstream research in control theory simply because stochastic noises are often inevitable in system modeling. Compared with the fruitful results on deterministic SFNSs, the corresponding results on stochastic SFNSs have been relatively fewer. So far, some efforts have been made to solve the control problem for the stochastic nonlinear systems by using the quartic Lyapunov function in combination with the Itô's differentiation rule. For example, an interesting inverse optimal control scheme [8] has been proposed to ensure the asymptotic stability in probability for SFNSs with stochastic disturbances. By applying the backstepping procedure and the stochastic small-gain theorem, an output-feedback adaptive controller has been developed in [40] for stochastic SFNSs in the presence of unknown parameters. By combining function approximation and backstepping techniques, an adaptive tracking control scheme has been put forward for a class of stochastic SFNSs with unknown functions [2]. Subsequently, some adaptive control schemes have been developed to achieve the closed-loop stability in probability for stochastic SFNSs exhibiting certain system constraints [35].

It should be pointed out that almost all existing results concerning SFNSs have been exclusively on the continuoustime case. In contrast to the rich body of literature available on continuous-time systems, only a few results have been obtained for discrete-time SFNSs despite their importance in 
modeling nowadays popular communication-based networked systems. In fact, many dedicated mathematical tools for continuous-time SFNSs cannot be directly exploited in the discrete-time case [11], [44], [47]. For example, the stability analysis based on traditional Lyapunov functions becomes extremely intractable for discrete-time SFNSs because the difference of the Lyapunov function in the discrete-time setting is inherently nonlinear, which makes it very difficult to design an appropriate controller to compensate/eliminate system uncertainties. In particular, if we were to directly apply the backstepping procedure to discrete-time SFNSs, the future state information is likely to appear in the controller, which would violate the local causality and lead to the infeasibility of the control scheme [11]. To resolve the causality contradiction issue, a seminal control scheme has been proposed in [44] for discrete-time SFNSs with unknown parameters. The presented method [44] is suitable for those systems that can be transformed into the parametric strict-feedback form [47]. By the $n$-step-ahead predictor method and function approximation technology, a systematic design framework [11], [13] has been proposed to solve the control problem for more general discrete-time SFNSs, where the basic idea is to convert the discrete-time SFNSs into the $n$-step-ahead predictor model and then design the control scheme for such a transformed system. With help of the elegant $n$-step-ahead predictor method, some extensions have been developed for more general nonlinear systems with different phenomena including the non-affine form [34], [41], unknown control directions [42], and input nonlinearities [24].

Up to now, all the aforementioned results for discrete-time SFNSs have been limited to deterministic systems without consideration of stochastic noises. As a matter of fact, many practical systems are subject to stochastic disturbances due to random abrupt variations such as sudden environmental changes, component failures, and changing subsystem interconnections [10], [14], [36]. As a consequence, it is of both theoretical significance and practical importance to study the control problem for discrete-time SFNSs under stochastic noises. From a methodological viewpoint, unlike the continuoustime case, there is a lack of appropriate mathematical tools capable of analyzing how the stochastic phenomenon affect the dynamical behaviors of discrete-time stochastic SFNSs. Recently, an initial effort has been made in [29] to address the stabilization problem for a class of discrete-time outputdependent nonlinear stochastic system with additive noises (that is independent of states), and some interesting results have been obtained under certain rather stringent assumptions (e.g. perfect system model and output-dependent nonlinear functions). On the other hand, it is often the case in practice that the stochastic noises encountered exert influence on system states [25]. Such kind of noises is referred to as the multiplicative noises (also called Itô-type noises) that not only affect system stability but also complicate the corresponding dynamic analysis [9], [10], [46].

So far, there have been very few (if not none) available results on the control problem for discrete-time SFNSs subject to multiplicative noises due probably to the technical challenges identified as follows. First, within the usual Lyapunovstability-based framework, the multiplicative noise enters into the difference of a Lyapunov function that leads to an additional state-dependent term. Recall that a Lyapunov function is normally constructed based on the controlled error. In this case, it becomes fundamentally difficult to obtain a stability criterion (in probability) with both system states and controlled errors appeared in the difference of the Lyapunov function. To overcome such a difficulty, some dedicated techniques have to be developed to characterize the system states by the controlled errors without inducing much conservatism. Another challenge stems from the unknown nonlinear modeling dynamics that cannot be simply approximated by the neural network (NN) in each step of backstepping because of the recursively accumulated approximation errors. Note that, as such errors become larger, it is more difficult to represent the system states by the controller errors which would invalidate the backstepping-based design in the sense of multiplicative noises. As such, the main motivation of this paper is to tackle the identified challenges by establishing a novel yet feasible control framework.

Motivated by the discussions made above, we will launch a major study on the stability analysis and controller design issues for a class of discrete-time SFNSs subject to the multiplicative noises. The noises, which are dependent on all system states, are driven by the Gaussian white noise sequence. Such kind of multiplicative noises is, for the first time, discussed in the control issue of discrete-time SFNSs. By combining the backstepping procedure with the Lyapunov stability theory, a novel backstepping control scheme is developed to provide a sufficient condition on the mean-square stability of the closed-loop systems, and the corresponding results are further extended to the systems with unknown modeling dynamics approximated by the radial basis function (RBF) NNs. The main contributions of the paper are highlighted as follows.

1) A novel backstepping-based control framework, which is essentially different from the $n$-step-ahead predictor method, is proposed to successfully establish the relationship between the system states and the controlled errors so as to facilitate the stability analysis with respect to the multiplicative noises.

2) The proposed new framework is based on the original stochastic controlled system (rather than the transformed $n$-step-ahead predictor model), which effectively avoids the effects on the closed-loop stability from the prediction errors. Meanwhile, the causality contradiction is also overcome by using the new variable substitution technology to obtain the future information.

3) A novel adaptive neural control scheme is developed by using only one neural approximator. Such a scheme not only avoids the delays of neural weight updating law caused by the classical $n$-step-ahead predictor model but also simplifies the algorithm implementation, thereby improving the transient-state performance and reducing the computational burden.

\section{Problem Formulation AND PRELIMINARIES}

Consider the following discrete-time strict-feedback nonlinear system with multiplicative noise

$\left\{\begin{aligned} x_{i}(k+1) & =g_{i}\left(\bar{x}_{i}(k)\right) x_{i+1}(k)+f_{i}\left(\bar{x}_{i}(k)\right), 1 \leq i \leq n-1 \\ x_{n}(k+1) & =g_{n}\left(\bar{x}_{n}(k)\right) u(k)+f_{n}\left(\bar{x}_{n}(k)\right)+h\left(\bar{x}_{n}(k)\right) \omega(k) \\ y(k) & =x_{1}(k)\end{aligned}\right.$ 
where $\bar{x}_{i}(k)=\left[x_{1}(k), x_{2}(k), \cdots, x_{i}(k)\right]^{T} \in \mathbb{R}^{i}, i=$ $1, \cdots, n, y(k) \in \mathbb{R}$ and $u(k) \in \mathbb{R}$ denote the state vector, the system output and the control input, respectively. $\omega(k) \in \mathbb{R}$ is a Gaussian white noise sequence with statistical properties $\mathbb{E}(\omega(k))=0$ and $\mathbb{E}\left(\omega^{2}(k)\right)=1 . g_{i}\left(\bar{x}_{i}(k)\right) \in \mathbb{R}, f_{i}\left(\bar{x}_{i}(k)\right) \in$ $\mathbb{R}(i=1,2, \cdots, n)$ and $h\left(\bar{x}_{n}(k)\right) \in \mathbb{R}$ are smooth nonlinear functions with $f_{i}(0)=0, h(0)=0$.

For convenience, we introduces the following notations: $C:=D$ means that $D$ is denoted as $C$, and $\|$.$\| denotes the$ Euclidean norm of a vector, namely, $\left\|\bar{x}_{i}(k)\right\|=\sqrt{\bar{x}_{i}^{T}(k) \bar{x}_{i}(k)}$.

Assumption 1: The nonlinear functions $f_{i}\left(\bar{x}_{i}(k)\right) \in \mathbb{R}$ $(i=1,2, \cdots, n-1)$ and $h\left(\bar{x}_{n}(k)\right) \in \mathbb{R}$ satisfy the Lipschitz condition.

Assumption 2: the smooth nonlinear function $g_{i}\left(\bar{x}_{i}(k)\right)$ $(i=1,2, \cdots, n)$ satisfies the controllable condition $0<\underline{g}_{i} \leq$ $\left|g_{i}(\cdot)\right| \leq \bar{g}_{i}$, in which $\underline{g}_{i}$ and $\bar{g}_{i}$ are two positive constants. Without losing generality, $g_{i}(\cdot)(i=1,2, \cdots, n)$ is assumed to be positive in this paper.

The primary objective of this paper is to design a backstepping-based state-feedback controller for the system (1) satisfying Assumption 1 such that, in the presence of the multiplicative noises, all of the closed-loop states are exponentially mean-square (EMS) stable, and the non-causality problem resulting from backstepping design is simultaneously avoided.

Remark 1: It should be noticed that some elegant control schemes [11], [13], [24] have been developed for discretetime SFNSs with bounded disturbances that are assumed to be deterministic. In practice, however, the disturbance often occurs randomly due to sudden environment changes that rely on system states, which gives rise to the multiplicative noise. It should be pointed out that, in the presence of multiplicative noise $h\left(\bar{x}_{n}(k)\right) \omega(k)$, the control system (1) is inherently stochastic and is therefore impossible to be transformed into the $n$-step ahead predictor model for solving the non-causality problem by the traditional predictor methods [11], [13], [24]. As such, a novel control framework has to be sought to overcome the essential difficulties caused by multiplicative noise $h\left(\bar{x}_{n}(k)\right) \omega(k)$.

Notice that nonlinear functions can be approximated by many function approximators such as polynomials, artificial NNs and fuzzy logic systems, where the NN approximators integrate well with the Lyapunov-stability-based nonlinear control framework. In this paper, the following RBF NN is employed as a function approximator:

$$
f_{n n}(Z(k))=W^{T} S(Z(k))
$$

where $Z(k) \in \Omega_{Z} \subset \mathbb{R}^{m}$ is the input of $\mathrm{RBF} \mathrm{N}$ $\mathrm{N}, \Omega_{Z}$ is a compact set, $W=\left[W_{1}, W_{2}, \cdots, W_{q}\right]^{T} \in$ $\mathbb{R}^{q}$ is the adjustable weight vector with $q>1$ being the node number of hidden layer, and $S(Z(k))=$ $\left[S_{1}(Z(k)), S_{2}(Z(k)), \cdots, S_{q}(Z(k))\right]^{T} \in \mathbb{R}^{q}$ is the basis function vector. In this paper, $S_{i}(Z(k))$ is selected as the Gaussian function $S_{i}(Z(k))=\exp \left[\frac{-\left(Z(k)-\varsigma_{i}\right)^{T}\left(Z(k)-\varsigma_{i}\right)}{r^{2}}\right]$, where $i=1, \cdots, q, \varsigma_{i} \in \mathbb{R}^{m}$ and $r \in \mathbb{R}$ are the center and width of the Gaussian function, respectively.

As shown in [30], the RBF NN (2) with sufficiently large node number $q$ can approximate any smooth function $f(Z(k))$, $\mathbb{R}^{m} \rightarrow \mathbb{R}$, to any accuracy over a compact set $\Omega_{Z} \subset \mathbb{R}^{m}$ :

$$
f(Z(k))=W^{* T} S(Z(k))+\delta(Z(k)), \forall Z(k) \in \Omega_{Z}
$$

where $W^{*}$ is an optimal constant weight vector, $\delta(Z(k))$ is the approximation error and satisfies $\delta(Z(k)) \leq \varepsilon$ with $\varepsilon$ being an arbitrarily small constant.

In order to verify the stability of the closed-loop system with multiplicative noise, a sufficient condition on the mean-square stability [43] is recalled as follows.

Lemma 1: [43] Define $\eta(k)=\left[\eta_{1}(k), \eta_{2}(k), \cdots\right.$, $\left.\eta_{n}(k)\right]^{T} \in \mathbb{R}^{n}$ and let $V(\eta(k))$ be a Lyapunov function. If there exist real scalars $\lambda_{1}>0, \lambda_{2}>0, \rho \geq 0$ and $0<\psi<1$ such that

$$
\lambda_{1}\|\eta(k)\|^{2} \leq V(\eta(k)) \leq \lambda_{2}\|\eta(k)\|^{2}
$$

and

$$
\mathbb{E}\{V(\eta(k+1)) \mid \eta(k)\}-V(\eta(k)) \leq-\psi V(\eta(k))+\rho
$$

then the sequence $\eta(k)$ is EMS stable and satisfies

$$
\mathbb{E}\left\{\|\eta(k)\|^{2}\right\} \leq \frac{\lambda_{2}}{\lambda_{1}}\|\eta(0)\|^{2}(1-\psi)^{k}+\frac{\rho}{\lambda_{1} \psi}
$$

where $\eta(0) \in \mathbb{R}^{n}$ is the given initial condition.

From Lemma 1, the sequence $\eta(k)$ is EMS stable if $\rho=0$, and $\eta(k)$ is EMS bounded if $\rho>0$.

\section{BACKSTEPPING-BASED CONTROL FRAMEWORK FOR EXACTLY KNOWN MODEL}

For clarity purposes, this section focuses on the case that the system model (1) is exactly known, that is, the system dynamics $f_{i}\left(\bar{x}_{i}(k)\right) \in \mathbb{R}$ and $g_{i}\left(\bar{x}_{i}(k)\right) \in \mathbb{R}$ in (1) are known for $i=1,2, \cdots, n$. It should be pointed out that the presence of the multiplicative noise $h\left(\bar{x}_{n}(k)\right) \omega(k)$ in (1) not only affects the stability but also complicates the establishment of the stability criteria for the closed-loop system. Furthermore, the causality contradiction constitutes another major obstacle encountered in the controller design of discrete-time SFNSs using the backstepping procedure. To deal with the stability and causality issues simultaneously, a novel control framework is proposed in this section for the system (1) with exact model information by combining the backstepping strategy and the variable substitution.

To start with, let us first introduce the following coordinate transformations:

$$
\left\{\begin{array}{l}
z_{1}(k)=x_{1}(k) \\
z_{i}(k)=x_{i}(k)-\alpha_{i-1}(k), i=2,3, \cdots, n
\end{array}\right.
$$

where the function $\alpha_{i-1}(k)$ is the virtual control law to be designed later. Based on the coordinate transformations (7), the following $n$-step recursive design procedure is used to derive the virtual control laws and the actual control law.

Step 1: Taking the error variable $z_{1}(k)=x_{1}(k)$ into consideration, its difference along $z_{2}(k)=x_{2}(k)-\alpha_{1}(k)$ is calculated as follows:

$$
\begin{aligned}
z_{1}(k+1) & =g_{1}\left(x_{1}(k)\right) x_{2}(k)+f_{1}\left(x_{1}(k)\right) \\
& =g_{1}\left(x_{1}(k)\right)\left[z_{2}(k)+\alpha_{1}(k)+\frac{f_{1}\left(x_{1}(k)\right)}{g_{1}\left(x_{1}(k)\right)}\right] .
\end{aligned}
$$

Constructing the virtual control law

$$
\alpha_{1}(k)=-\frac{f_{1}\left(x_{1}(k)\right)}{g_{1}\left(x_{1}(k)\right)}:=F_{1}\left(z_{1}(k)\right)
$$


we have

$$
z_{1}(k+1)=g_{1}\left(x_{1}(k)\right) z_{2}(k):=G_{1}\left(z_{1}(k)\right) z_{2}(k) .
$$

According to the Assumption 1 and Assumption 2, it is not difficult for one to figure out that $F_{1}\left(z_{1}(k)\right)$ also satisfies the Lipschitz condition.

Step 2: Noting $z_{2}(k)=x_{2}(k)-\alpha_{1}(k)$, its first difference along (1) and (7) is

$$
\begin{aligned}
z_{2}(k+1) & =g_{2}\left(\bar{x}_{2}(k)\right)\left[z_{3}(k)+\alpha_{2}(k)\right. \\
& \left.+\frac{f_{2}\left(\bar{x}_{2}(k)\right)-\alpha_{1}(k+1)}{g_{2}\left(\bar{x}_{2}(k)\right)}\right] .
\end{aligned}
$$

To overcome the causality contradiction caused by $\alpha_{1}(k+$ $1)$, the term $\alpha_{1}(k+1)$ is characterized along (9) and (10) as

$$
\alpha_{1}(k+1)=F_{1}\left(z_{1}(k+1)\right)=F_{1}\left(G_{1}\left(z_{1}(k)\right) z_{2}(k)\right)
$$

Substituting (12) into (11) gives

$$
\begin{aligned}
z_{2}(k+1) & =g_{2}\left(\bar{x}_{2}(k)\right)\left[z_{3}(k)+\alpha_{2}(k)\right. \\
& \left.+\frac{f_{2}\left(\bar{x}_{2}(k)\right)-F_{1}\left(G_{1}\left(z_{1}(k)\right) z_{2}(k)\right)}{g_{2}\left(\bar{x}_{2}(k)\right)}\right] .
\end{aligned}
$$

Next, constructing the second virtual control

$$
\alpha_{2}(k)=-\frac{f_{2}\left(\bar{x}_{2}(k)\right)-F_{1}\left(G_{1}\left(z_{1}(k)\right) z_{2}(k)\right)}{g_{2}\left(\bar{x}_{2}(k)\right)}
$$

yields

$$
\begin{aligned}
z_{2}(k+1) & =g_{2}\left(\bar{x}_{2}(k)\right) z_{3}(k) \\
& =g_{2}\left(z_{1}(k), z_{2}(k)+\alpha_{1}(k)\right) z_{3}(k) \\
& :=G_{2}\left(\bar{z}_{2}(k)\right) z_{3}(k) .
\end{aligned}
$$

To facilitate the stability analysis, we will show that the virtual control $\alpha_{2}(k)$ can be characterized as a function of error variables $z_{1}(k)$ and $z_{2}(k)$. Firstly, noting $z_{1}(k)=x_{1}(k)$ and according to (9), we have $\alpha_{1}(k)=F_{1}\left(z_{1}(k)\right)$. It then follows from $x_{2}(k)=z_{2}(k)+\alpha_{1}(k)$ that $x_{2}(k)=z_{2}(k)+F_{1}\left(z_{1}(k)\right)$. Therefore, $f_{2}\left(\bar{x}_{2}(k)\right)$ as well as $g_{2}\left(\bar{x}_{2}(k)\right.$ can be regarded as a function of the variables $z_{1}(k)$ and $z_{2}(k)$. Subsequently, the virtual control $\alpha_{2}(k)$ is rewritten as

$$
\begin{aligned}
\alpha_{2}(k) & =-\frac{f_{2}\left(z_{1}(k), z_{2}(k)+F_{1}\left(z_{1}(k)\right)\right)-F_{1}\left(G_{1}\left(z_{1}(k)\right) z_{2}(k)\right)}{g_{2}\left(z_{1}(k), z_{2}(k)+F_{1}\left(z_{1}(k)\right)\right)} \\
& :=F_{2}\left(\bar{z}_{2}(k)\right)
\end{aligned}
$$

where $\bar{z}_{2}(k)=\left[z_{1}(k), z_{2}(k)\right]^{T} \in \mathbb{R}^{2}$. According to Assumption 1 and Assumption 2, it can be concluded that the function $F_{2}\left(\bar{z}_{2}(k)\right)$ satisfies the Lipschitz condition.

Step $i(3 \leq i \leq n-1)$ : Defining $z_{i}(k)=x_{i}(k)-\alpha_{i-1}(k)$, and using (1), we have

$$
\begin{aligned}
z_{i}(k+1) & =g_{i}\left(\bar{x}_{i}(k)\right)\left[z_{i+1}(k)+\alpha_{i}(k)\right. \\
& \left.+\frac{f_{i}\left(\bar{x}_{i}(k)\right)-\alpha_{i-1}(k+1)}{g_{i}\left(\bar{x}_{i}(k)\right)}\right] .
\end{aligned}
$$

Applying the similar analysis as in Step 2 and according to (16), we obtain $\alpha_{i-1}(k)$ by a recursive design as follows:

$$
\begin{aligned}
\alpha_{i-1}(k)= & -f_{i-1}\left(\bar{x}_{i-1}(k)\right) / g_{i}\left(\bar{x}_{i-1}(k)\right) \\
& \left.+F_{i-2}\left(G_{1}\left(z_{1}(k)\right) z_{2}(k)\right), G_{2}\left(\bar{z}_{2}(k)\right) z_{3}(k)\right),
\end{aligned}
$$

$$
\begin{aligned}
& \left.\cdots, G_{i-2}\left(\bar{z}_{i-2}(k)\right) z_{i-1}(k)\right) / g_{i}\left(\bar{x}_{i-1}(k)\right) \\
:=F_{i-1}\left(\bar{z}_{i-1}(k)\right) & \text { (18) }
\end{aligned}
$$

where

$$
\bar{z}_{i-1}(k)=\left[z_{1}(k), z_{2}(k), \cdots, z_{i-1}(k)\right]^{T} \in \mathbb{R}^{i-1} .
$$

To this end, the term $\alpha_{i-1}(k+1)$ in (17) along (18) is expressed as

$$
\alpha_{i-1}(k+1)=F_{i-1}\left(\bar{z}_{i-1}(k+1)\right) .
$$

From (10) and (15), it can be recursively obtained that $z_{j-1}(k+1)=G_{j-1}\left(\bar{z}_{j-1}\right) z_{j}(k), j=2,3, \cdots, i$. Therefore, $\bar{z}_{i-1}(k+1)$ can be further characterized as the function of $\bar{z}_{i}(k)$. Based on the above analysis, the term $\alpha_{i-1}(k+1)$ including the future information $\bar{z}_{i-1}(k+1)$ can be rewritten using the current error variables $\bar{z}_{i}(k)$, which is shown specifically as follows:

$$
\begin{gathered}
\alpha_{i-1}(k+1)=F_{i-1}\left(G_{1}\left(z_{1}(k)\right) z_{2}(k), G_{2}\left(\bar{z}_{2}(k)\right) z_{3}(k),\right. \\
\left.\cdots, G_{i-1}\left(\bar{z}_{i-1}\right) z_{i}(k)\right) \\
:=F_{i-1}\left(\overline{G_{i-1}\left(\bar{z}_{i-1}\right) z_{i}(k)}\right)
\end{gathered}
$$

where $\overline{G_{i-1}\left(\bar{z}_{i-1}\right) z_{i}(k)}=\left[G_{1}\left(z_{1}(k)\right) z_{2}(k), \cdots\right.$, $\left.G_{i-1}\left(\bar{z}_{i-1}\right) z_{i}(k)\right]^{T}, \quad G_{j}\left(\bar{z}_{j}(k)\right)=g_{j}\left(z_{1}(k), z_{2}(k)+\right.$ $\left.\alpha_{1}(k), \cdots, z_{j}(k)+\alpha_{j-1}(k)\right), j=1,2, \cdots, i-1$.

Subsequently, constructing the $i$-th virtual control law

$$
\alpha_{i}(k)=-\frac{f_{i}\left(\bar{x}_{i}(k)\right)-F_{i-1}\left(\overline{G_{i-1}\left(\bar{z}_{i-1}\right) z_{i}(k)}\right)}{g_{i}\left(\bar{x}_{i}(k)\right)}
$$

we have

$$
z_{i}(k+1)=g_{i}\left(\bar{x}_{i}(k)\right) z_{i+1}(k):=G_{i}\left(\bar{z}_{i}(k)\right) z_{i+1}(k)
$$

Similarly, noticing $x_{i}(k)=z_{i}(k)+\alpha_{i-1}(k)$ with $\alpha_{i-1}(k+1)$ described by (20), it can be obtained that the virtual control $\alpha_{i}(k)$ in (22) can be characterized as a function of error variables $z_{j}(k), j=1,2, \cdots, i$. As a result, we rewrite $\alpha_{i}(k)$ as follows:

$$
\alpha_{i}(k)=F_{i}\left(\bar{z}_{i}(k)\right)
$$

where $F_{i}\left(\bar{z}_{i}(k)\right)$ denotes

$$
\begin{aligned}
F_{i}\left(\bar{z}_{i}(k)\right)=- & {\left[f _ { i } \left(z_{1}(k), z_{2}(k)+F_{1}\left(z_{1}(k)\right), \cdots, z_{i}(k)\right.\right.} \\
& \left.\left.+F_{i-1}\left(\bar{z}_{i-1}(k)\right)\right)-F_{i-1}\left(\overline{G_{i-1}\left(\bar{z}_{i-1}\right) z_{i}(k)}\right)\right] / \\
g_{i}\left(z_{1}(k), z_{2}(k)+F_{1}\left(z_{1}(k)\right), \cdots, z_{i}(k)\right. & \\
& \left.+F_{i-1}\left(\bar{z}_{i-1}(k)\right)\right) .
\end{aligned}
$$

Step $n$ : For $z_{n}(k)=x_{n}(k)-\alpha_{n-1}(k)$, its difference is

$$
\begin{aligned}
z_{n}(k+1)= & g_{n}\left(\bar{x}_{n}(k)\right)\left[u(k)+\frac{f_{n}\left(\bar{x}_{n}(k)\right)-\alpha_{n-1}(k+1)}{g_{n}\left(\bar{x}_{n}(k)\right)}\right] \\
& +h\left(\bar{x}_{n}(k)\right) \omega(k) .
\end{aligned}
$$

From (23) and (24), the term $\alpha_{n-1}(k+1)$ is expressed as

$$
\begin{aligned}
\alpha_{n-1}(k+1) & =F_{n-1}\left(\bar{z}_{n-1}(k+1)\right) \\
& =F_{n-1}\left(\overline{G_{i-1}\left(\bar{z}_{i-1}\right) z_{i}(k)}\right)
\end{aligned}
$$

where $F_{n-1}\left(\bar{z}_{n-1}(k)\right)$ is given in (25) with $i=$ $n-1$, and $\overline{G_{n-1}\left(\bar{z}_{n-1}\right) z_{n}(k)}=\left[G_{1}\left(z_{1}(k)\right) z_{2}(k), \cdots\right.$, $\left.G_{i-1}\left(\bar{z}_{n-1}\right) z_{n}(k)\right]^{T}$. Substituting (27) into (26) gives

$$
z_{n}(k+1)=g_{n}\left(\bar{x}_{n}(k)\right)\left[u(k)+\frac{f_{n}\left(\bar{x}_{n}(k)\right)}{g_{n}\left(\bar{x}_{n}(k)\right)}\right.
$$




$$
\left.-\frac{F_{n-1}\left(\overline{G_{i-1}\left(\bar{z}_{i-1}\right) z_{i}(k)}\right)}{g_{n}\left(\bar{x}_{n}(k)\right)}\right]+h\left(\bar{x}_{n}(k)\right) \omega(k) .
$$

For clarity, the ideal actual controller $u(k)$ is denoted as $u^{*}(k)$, that is, $u(k):=u^{*}(k)$ when the system model (1) is exactly known. Subsequently, constructing the ideal actual controller as follows:

$$
u^{*}(k)=\frac{-f_{n}\left(\bar{x}_{n}(k)\right)+F_{n-1}\left(\overline{G_{i-1}\left(\bar{z}_{i-1}\right) z_{i}(k)}\right)}{g_{n}\left(\bar{x}_{n}(k)\right)}
$$

we have

$$
z_{n}(k+1)=h\left(\bar{x}_{n}(k)\right) \omega(k) .
$$

Up to now, we have completed the backstepping-based controller design.

Lemma 2: Consider the coordinate transformation (7), the virtual control law (9), (16) and (24). Under the Assumption 1 and Assumption 2, we have

$$
\left|x_{i}(k)\right| \leq\left|z_{i}(k)\right|+L_{F, i-1}\left\|\bar{z}_{i-1}(k)\right\|, i=2,3, \cdots, n
$$

where $L_{F_{i-1}}$ is a Lipschitz constant of the nonlinear function $F_{i}\left(\bar{z}_{i}(k)\right)$ in $(25)$.

Proof: From the coordinate transformation (7), it is obtained that $x_{1}(k)=z_{1}(k), x_{i}(k)=z_{i}(k)+\alpha_{i-1}(k)$. The virtual control law $\alpha_{i}(k)$ is characterized as $F_{i}\left(\bar{z}_{i}(k)\right)$. According to the equation (9), (16) and (24), it is easy to figure out that $F_{i}(0)=0(i=1,2, \cdots, n-1)$. For $F_{1}\left(z_{1}(k)\right)$, one has the following property holds under the Assumption 1 and Assumption 2.

$$
\left|F_{1}\left(z_{1}(k)\right)\right|=\left|\frac{f_{1}\left(z_{1}(k)\right)}{g_{1}\left(z_{1}(k)\right)}\right| \leq \frac{\left|f_{1}\left(z_{1}(k)\right)\right|}{\underline{g}_{i}} \leq L_{F_{1}}\left|z_{1}(k)\right|
$$

where $L_{F_{1}}=L_{f_{1}} / \underline{g}_{1}, L_{f_{1}}$ is the Lipschitz constant of the nonlinear function $f_{1}\left(z_{1}(k)\right)$. It can be concluded from (9) and (32) that $F_{1}\left(z_{1}(k)\right)$ satisfies the Lipschitz condition. Referring to the definition of $F_{2}\left(\bar{z}_{2}(k)\right)$, one has

$$
\begin{aligned}
\left|F_{2}\left(\bar{z}_{2}(k)\right)\right| \leq & \frac{\left|f_{2}\left(z_{1}(k), z_{2}(k)+F_{1}\left(z_{1}(k)\right)\right)\right|}{\underline{g}_{2}} \\
& +\frac{\left|F_{1}\left(g_{1}\left(z_{1}(k)\right) z_{2}(k)\right)\right|}{\underline{g}_{2}} .
\end{aligned}
$$

Since $f_{2}(\cdot)$ and $F_{1}(\cdot)$ satisfy the Lipschitz condition, it is finally obtained that.

$$
\left|F_{2}\left(\bar{z}_{2}(k)\right)\right| \leq L_{F_{2}}\left\|\bar{z}_{2}(k)\right\|
$$

where $L_{F_{2}}=\max \left\{L_{f_{2}} \sqrt{\max \left\{1+2 L_{F_{1}}^{2}, 2\right\}}, L_{F_{1}} \bar{g}_{1}\right\} / \underline{g}_{2}$, $L_{f_{2}}$ is the Lipschitz constant of $f_{2}(\cdot)$. Thus, $F_{2}\left(\bar{z}_{2}(k)\right)$ also satisfies the Lipschitz condition. Recursively, one has

$$
\left|F_{i}\left(\bar{z}_{i}(k)\right)\right| \leq L_{F_{i}}\left\|\bar{z}_{i}(k)\right\|
$$

where

$$
\begin{aligned}
L_{F_{i}}=\max & \left\{L_{F_{i-1}} \max \left\{\bar{g}_{1}, \cdots, \bar{g}_{i-1}\right\},\right. \\
& \left.\frac{L_{f_{i}}}{\underline{g}_{i}} \sqrt{\max \left\{\theta_{1}, \theta_{2}, \cdots, \theta_{i}\right\}}\right\},
\end{aligned}
$$

$$
\begin{aligned}
& \theta_{1}=1+2 \sum_{j=1}^{i-1} L_{F_{j}}^{2}, \quad \theta_{m}=2+2 \sum_{j=m}^{i-1} L_{F_{j}}^{2}, \\
& i=2,3, \cdots, n-1 ; \quad m=2,3, \cdots, i
\end{aligned}
$$

with $L_{F_{i}}$ being a Lipschitz constant of the nonlinear function $F_{i}\left(\bar{z}_{i}(k)\right)$. Consequently, the relationship between $x_{i}(k)$ and $z_{i}(k)$ is derived as

$$
\begin{aligned}
\left|x_{i}(k)\right| & =\left|z_{i}(k)+\alpha_{i-1}(k)\right| \\
& \leq\left|z_{i}(k)\right|+L_{F_{i-1}}\left\|\bar{z}_{i-1}(k)\right\|
\end{aligned}
$$

where $i=2,3, \cdots, n-1$.

Theorem 1: Consider the closed-loop system consisting of the discrete-time strict-feedback nonlinear systems (1) with multiplicative noise $h\left(\bar{x}_{n}(k)\right) \omega(k)$, Assumptions 1-2, the virtual control law (22) and the actual controller (29). For any given initial condition, the closed-loop system is EMS stable if there exist constants $p_{i}>0$ and $0<\psi<1$ such that the following conditions hold

$$
p_{i}-p_{i-1} \bar{g}_{i-1}^{2}-p_{n} L_{i}-p_{i} \psi \geq 0, \quad i=1,2, \cdots, n
$$

where $p_{0}=0, g_{0}=0, \bar{g}_{i-1}$ is the upper bound of the nonlinear function $g_{i-1}\left(\bar{x}_{i-1}(k)\right)$, and $L_{i}>0$ is to be defined later which relies on Lipschitz constants of nonlinear functions $h\left(\bar{x}_{n}(k)\right)$ and $f_{i}\left(\bar{x}_{i}(k)\right)$ with $i=1,2, \cdots, n-1$.

Proof: Construct the following Lyapunov function

$$
V(k)=\sum_{i=1}^{n} p_{i} z_{i}^{2}(k)
$$

where $p_{i}(i=1,2, \cdots, n)$ is a positive design constant.

By defining $\lambda_{1}=\min \left\{p_{1}, p_{2}, \cdots, p_{n}\right\}$ and $\lambda_{2}=$ $\max \left\{p_{1}, p_{2}, \cdots, p_{n}\right\}$, it is easily obtained that $\lambda_{1}\|z(k)\|^{2} \leq$ $V(k) \leq \lambda_{2}\|z(k)\|^{2}$, which means the chosen Lyapunov function (37) satisfies Lemma 1. Then, the difference of (37) along (10), (15), (23) and (30) is given by

$$
\begin{aligned}
\Delta V(k)= & \mathbb{E}\{V(k+1) \mid z(k)\}-V(k) \\
= & -\sum_{i=1}^{n}\left(p_{i}-p_{i-1} g_{i-1}^{2}\left(\bar{x}_{i-1}(k)\right)\right) z_{i}^{2}(k) \\
& +\mathbb{E}\left\{p_{n}\left[h\left(\bar{x}_{n}(k)\right) \omega(k)\right]^{2}\right\} \\
\leq & p_{n} h^{2}\left(\bar{x}_{n}(k)\right)-\sum_{i=1}^{n}\left(p_{i}-p_{i-1} \bar{g}_{i-1}^{2}\right) z_{i}^{2}(k)
\end{aligned}
$$

where $p_{0}=0$.

It is seen from (38) that the non-zero unbounded function $h\left(\bar{x}_{n}(k)\right)$ makes the stability analysis for (1) extremely difficult. To overcome such a difficulty, the key issue is to characterize the functions $h\left(\bar{x}_{n}(k)\right)$ by using the error variables $z_{i}(k)(i=1,2, \cdots, n)$. Noticing $h(0)=0$ and Assumption 1, there exists a positive constant $L_{h}$ such that

$$
\left|h\left(\bar{x}_{n}(k)\right)-h(0)\right| \leq L_{h}\left\|\bar{x}_{n}(k)\right\| .
$$

Substituting (40) into (38) and referring to the Lemma 2, we have

$$
\begin{aligned}
\Delta V(k) & \leq p_{n} L_{h}^{2} \sum_{i=1}^{n} x_{i}^{2}(k)-\sum_{i=1}^{n}\left(p_{i}-p_{i-1} \bar{g}_{i-1}^{2}\right) z_{i}^{2}(k) \\
& \leq-\psi V(k)-\sum_{i=1}^{n}\left(p_{i}-p_{i-1} \bar{g}_{i-1}^{2}\right.
\end{aligned}
$$




$$
\left.-p_{n} L_{i}-p_{i} \psi\right) z_{i}^{2}(k)
$$

where $\psi$ is a constant satisfying $0<\psi<1, L_{1}=L_{h}^{2}(1+$ $\left.2 \sum_{j=1}^{n-1} L_{F_{j}}^{2}\right), L_{i}=2 L_{h}^{2}\left(1+\sum_{j=i}^{n-1} L_{F_{j}}^{2}\right), i=2,3, \cdots, n-1$, and $L_{n}=2 L_{h}^{2}$. It is clearly shown from (41) that, if the condition (36) holds, then (41) is rewritten as

$$
\Delta V(k)=\mathbb{E}\{V(k+1) \mid z(k)\}-V(k) \leq-\psi V(k) .
$$

According to Lemma 1, we can conclude from (42) that the closed-loop system is EMS stable.

Remark 2: Theorem 1 provides a sufficient condition for the mean-square stability of discrete-time SFNSs (1) with multiplicative noise $h\left(\bar{x}_{n}(k)\right) \omega(k)$. It is worth pointing out that the proposed method is quite different from the existing ones used in [11], [13], [24] for systems without multiplicative noises. In this paper, some specific efforts have been devoted to deal with the difficulties in stability analysis caused by the multiplicative noise $h\left(\bar{x}_{n}(k)\right) \omega(k)$. Firstly, the virtual control law $\alpha_{i}(k)$ is recursively designed based on the original system (1) rather than the traditional predictor model. By using the variable substitution, the virtual control law $\alpha_{i}(k)$ is expressed in (24) as a function of errors $z_{j}(k), j=1,2, \cdots, i$. By combining the coordinate transformations (7), Assumption 1 and Lemma 2, the system state $\left|x_{i}(k)\right|$ is bounded by a linear combination of $\left\|z_{j}(k)\right\|, j=1,2, \cdots, i$. As a result, the term $L_{h}^{2} \sum_{i=1}^{n} x_{i}^{2}(k)$ resulting from the multiplicative noise $h\left(\bar{x}_{n}(k)\right) \omega(k)$ can be bounded by $\sum_{i=1}^{n} L_{i} z_{i}^{2}(k)$ in (41). Based on these dedicated efforts mentioned above, the stability condition (36) is derived to ensure that the discrete-time SFNSs (1) subject to multiplicative noises is EMS stable. It should be noticed that the key idea of our developed approach is to make the system state $x_{i}(k)$ bounded by the errors $z_{j}(k)$, $j=1,2, \cdots, i$.

Notice that the stability condition (36) can be simplified and Assumption 1 can be simultaneously relaxed if the multiplicative noise in system (1) is replaced with the following three cases: the special multiplicative noise $h\left(x_{1}(k)\right) \omega(k)$, the additive noise $\omega(k)$, or the deterministic bounded external disturbance $d(t)$. Based on the proof of Theorem 1, it is easy to obtain the following two corollaries.

Corollary 1: Consider the system (1) subject to the multiplicative noise $h\left(x_{1}(k)\right) \omega(k)$, in which $f_{i}\left(\bar{x}_{i}(k)\right) \quad(i=$ $1,2, \cdots, n)$ are not required to satisfy the Lipschitz condition. By designing the same virtual control laws (22) and the actual controller (29) as in Theorem 1, for any bounded initial condition, the closed-loop system is EMS stable if $h\left(x_{1}(k)\right)$ satisfies the Lipschitz condition and the constants are appropriately chosen such that

$$
p_{1}-p_{n} L^{2}-p_{i} \psi>0, p_{i}-p_{i-1} \bar{g}_{i-1}^{2}-p_{i} \psi \geq 0
$$

where $i=2,3, \cdots, n, p_{i}>0,0<\psi<1, L>0$ is the Lipschitz constant of the function $h\left(x_{1}(k)\right), \bar{g}_{i-1}$ is the upper bound of the nonlinear function $g_{i-1}\left(\bar{x}_{i-1}(k)\right)$.

Corollary 2: Consider the system (1) by replacing the multiplicative noise $h\left(\bar{x}_{n}(k)\right) \omega(k)$ with the additive noise $\omega(k)$ (or bounded external disturbance $d(t)$ ), in which $f_{i}\left(\bar{x}_{i}(k)\right.$ ) $(i=1,2, \cdots, n)$ are not required to satisfy the Lipschitz condition. By designing the same virtual control laws (22) and the actual controller (29) as in Theorem 1, for any bounded initial condition, the closed-loop system is EMS stable (or uniformly ultimately bounded) if the following conditions are satisfied

$$
p_{i}-p_{i-1} \bar{g}_{i-1}^{2}-p_{i} \psi \geq 0, \quad i=1,2, \cdots, n
$$

where $p_{0}=g_{0}=0, p_{i}>0(2, \cdots, n), 0<\psi<1$, and $\bar{g}_{i-1}$ is the upper bound of the nonlinear function $g_{i-1}\left(\bar{x}_{i-1}(k)\right)$.

\section{BACKStepping-BAsed AdAptive Neural Control DESIGN FOR NONLINEAR MODELING UNCERTAINTIES}

In this section, we will extend the results obtained in Section III to systems with modeling uncertainties within the same framework in order to better reflect the engineering practice.

Assumption 3: The system dynamics $f_{i}\left(\bar{x}_{i}(k)\right) \in \mathbb{R}$ and $g_{i}\left(\bar{x}_{i}(k)\right) \in \mathbb{R}(i=1,2, \cdots, n)$ are unknown and smooth nonlinear functions.

From Assumption 3, the virtual control laws $\alpha_{i}(k)$ in (22) and the ideal actual control law $u^{*}(k)$ in (29) cannot be implemented since it contains the unknown dynamics of the system. Notice that the proposed control scheme in Section III makes all functions be passed down and lumped in the actual ideal controller $u^{*}(k)$ in (29). Based on such an analysis, we would only need one NN to approximate the ideal controller $u^{*}(k)$ consisting of $f_{n}\left(\bar{x}_{n}(k)\right), g_{n}\left(\bar{x}_{n}(k)\right)$ and $F_{n-1}\left(g_{1}\left(x_{1}(k)\right) z_{2}(k), \cdots, g_{n-1}\left(\bar{x}_{n-1}(k)\right) z_{n}(k)\right)$ if the controlled error variables $z_{i}(k)(i=2,3, \cdots, n)$ can be characterized by a function of system states $x_{i}(k) \quad(i=$ $1,2, \cdots, n)$. Noticing that $z_{2}(k)=x_{2}(k)-\alpha_{1}(k)$ and

$$
\alpha_{1}(k)=-f_{1}\left(x_{1}(k)\right) / g_{1}\left(x_{1}(k)\right)
$$

we have $z_{2}(k)=x_{2}(k)+f_{1}\left(x_{1}(k)\right) / g_{1}\left(x_{1}(k)\right)$. To facilitate the construction of a recursive formula between $z_{i}(k)$ and $x_{i}(k)$, let

$$
\phi_{2}\left(\bar{x}_{2}(k)\right)=x_{2}(k)+f_{1}\left(x_{1}(k)\right) / g_{1}\left(x_{1}(k)\right)
$$

which means $z_{2}(k)=\phi_{2}\left(\bar{x}_{2}(k)\right)$. Since $z_{3}(k)=x_{3}(k)-$ $\alpha_{2}(k)$ with $\alpha_{2}(k)$ given in (14), it can be derived that

$$
z_{3}(k)=x_{3}(k)+\frac{\left[f_{2}\left(\bar{x}_{2}(k)\right)-F_{1}\left(g_{1}\left(x_{1}(k)\right) \phi_{2}\left(\bar{x}_{2}(k)\right)\right)\right]}{g_{2}\left(\bar{x}_{2}(k)\right)} .
$$

Similarly, it follows from (7) and (22) that

$$
\begin{aligned}
z_{i}(k)= & x_{i}(k)+f_{i-1}\left(\bar{x}_{i-1}(k)\right) / g_{i-1}\left(\bar{x}_{i-1}(k)\right) \\
& -F_{i-2}\left(g_{1}\left(x_{1}(k)\right) \phi_{2}\left(\bar{x}_{2}(k)\right), g_{2}\left(\bar{x}_{2}(k)\right) \phi_{3}\left(\bar{x}_{3}(k)\right),\right. \\
& \left.\cdots, g_{i-2}\left(\bar{x}_{i-2}(k)\right) \phi_{i-1}\left(\bar{x}_{i-1}(k)\right)\right) / g_{i-1}\left(\bar{x}_{i-1}(k)\right) \\
:= & \phi_{i}\left(\bar{x}_{i}(k)\right)
\end{aligned}
$$

where $i=2, \cdots, n$, and $F_{0}()=$.0 .

From (45), the ideal controller $u^{*}(k)$ in (29) is rewritten as the function of all system states as follows:

$$
\begin{aligned}
u^{*}(k)=- & f_{n}\left(\bar{x}_{n}(k)\right) / g_{n}\left(\bar{x}_{n}(k)\right) \\
+ & F_{n-1}\left(g_{1}\left(x_{1}(k)\right) \phi_{2}\left(\bar{x}_{2}(k)\right), g_{2}\left(\bar{x}_{2}(k)\right) \phi_{3}\left(\bar{x}_{3}(k)\right),\right. \\
& \left.\cdots, g_{n-1}\left(\bar{x}_{n-1}\right) \phi_{n}\left(\bar{x}_{n}(k)\right)\right) / g_{n}\left(\bar{x}_{n}(k)\right) .
\end{aligned}
$$

It is easily seen from (46) that the ideal controller $u^{*}(k)$ in (29) can be regarded as a function of states $x_{i}(k)(i=1,2, \cdots, n)$ which does not rely on the virtual control law $\alpha_{i}(k)$. Moreover, according to the definition of $F_{n-1}($.$) in (25) and Assumption$ 3 , we know that $u^{*}(k)$ is a smooth function. As a result, $u^{*}(k)$ 
in (29) can be approximated to arbitrarily accuracy over a compact set $\Omega \subset \mathbb{R}^{n}$ by RBF NN (3) as follows:

$$
u^{*}(k)=W^{* T}(k) S\left(\bar{x}_{n}(k)\right)+\delta\left(\bar{x}_{n}(k)\right), \quad \forall \bar{x}_{n}(k) \in \Omega
$$

where $W^{*} \in \mathbb{R}^{q}$ is an ideal constant weight vector, $\delta\left(\bar{x}_{n}(k)\right)$ is the approximation error and satisfies $\delta\left(\bar{x}_{n}(k)\right) \leq \varepsilon$, and $\varepsilon$ is an arbitrarily small constant.

Substituting (47) into (28), we have

$$
\begin{aligned}
z_{n}(k+1)= & g_{n}\left(\bar{x}_{n}(k)\right)\left[u(k)-W^{* T} S\left(\bar{x}_{n}(k)\right)-\delta\left(\bar{x}_{n}(k)\right)\right] \\
& +h\left(\bar{x}_{n}(k)\right) \omega(k) .
\end{aligned}
$$

By designing the following adaptive neural controller

$$
u(k)=\hat{W}^{T}(k) S\left(\bar{x}_{n}(k)\right)
$$

we have

$$
\begin{aligned}
z_{n}(k+1)= & g_{n}\left(\bar{x}_{n}(k)\right)\left[\tilde{W}^{T}(k) S\left(\bar{x}_{n}(k)\right)-\delta\left(\bar{x}_{n}(k)\right)\right] \\
& +h\left(\bar{x}_{n}(k)\right) \omega(k)
\end{aligned}
$$

where $\hat{W}(k)$ is the estimate of the ideal neural weight $W^{*}$ and $\tilde{W}(k)=\hat{W}(k)-W^{*}$ is the estimate error.

In order to verify the mean-square boundedness of the neural weight estimate error $\tilde{W}(k)$, the neural weight update law is chosen as

$$
\hat{W}(k+1)=(1-\sigma) \hat{W}(k)+\frac{\gamma S\left(\bar{x}_{n}(k)\right) z_{1}(k)}{1+\left\|S\left(\bar{x}_{n}(k)\right)\right\|^{2} z_{1}^{2}(k)}
$$

where $\gamma>0$ is the design parameter and $\sigma>0$ is the modification coefficient. By using (50) and taking $\tilde{W}(k)=$ $\hat{W}(k)-W^{*}$ into account, we derive the neural weight estimate error dynamics as follows:

$$
\tilde{W}(k+1)=\tilde{W}(k)+\frac{\gamma S\left(\bar{x}_{n}(k)\right) z_{1}(k)}{1+\left\|S\left(\bar{x}_{n}(k)\right)\right\|^{2} z_{1}^{2}(k)}-\sigma \hat{W}(k) .
$$

Remark 3: From (46), the ideal controller $u^{*}(k)$ in (29) has been transformed into a function of all system states $\bar{x}_{n}(k)$ by recursively characterizing the error $z_{i}(k)$ as $\phi_{i}\left(\bar{x}_{i}(k)\right)$ in (45). As a result, the controller $u^{*}(k)$ in (46) is independent of the virtual control laws $\alpha_{i}(k)$ in (22). Therefore, only one $\mathrm{RBF} \mathrm{NN}$ in (47) is applied to approximate the ideal controller $u^{*}(k)$ in (46) since the virtual control laws $\alpha_{i}(k)$ are just used in the intermediate design process which does not need to be implemented in practice. Compared with existing methods with multiple neural approximators [11], [13], [24], the developed controller (48) and (50) can be easily implemented with significantly reduced computational burden.

Next, we first show that the neural weight estimate error is EMS bounded via the Lyapunov stability analysis.

Theorem 2: Consider the neural weight estimate error dynamics (51). Suppose that the neural weight $\hat{W}(0)$ is initialized in a compact set $\Omega$ and updated by (50). Then, the neural weight estimate error $\tilde{W}(k)$ is EMS bounded provided that design parameters satisfy $0<\gamma<1, \frac{1}{4 \varrho}<\sigma<0.5$, and $\varrho>1$.

Proof: Choose the following Lyapunov function

$$
V_{\tilde{W}}(k)=\tilde{W}^{T}(k) \tilde{W}(k)
$$

whose first difference along the weight estimate error dynamics (51) is given by

$$
\begin{aligned}
\Delta V_{\tilde{W}}(k)=\mathbb{E} & \left\{V_{\tilde{W}}(k+1) \mid \tilde{W}(k)\right\}-V_{\tilde{W}}(k) \\
=\mathbb{E} & \left\{\frac{2 \gamma \tilde{W}^{T}(k) S\left(\bar{x}_{n}(k)\right) z_{1}(k)}{1+\left\|S\left(\bar{x}_{n}(k)\right)\right\|^{2} z_{1}^{2}(k)}-2 \sigma \tilde{W}^{T}(k) \hat{W}(k)\right. \\
& +\frac{\gamma^{2}\left\|S\left(\bar{x}_{n}(k)\right)\right\|^{2} z_{1}^{2}(k)}{\left[1+\left\|S\left(\bar{x}_{n}(k)\right)\right\|^{2} z_{1}^{2}(k)\right]^{2}}+\sigma^{2}\|\hat{W}(k)\|^{2} \\
& \left.-\frac{2 \gamma \sigma \hat{W}^{T}(k) S\left(\bar{x}_{n}(k)\right) z_{1}(k)}{1+\left\|S\left(\bar{x}_{n}(k)\right)\right\|^{2} z_{1}^{2}(k)}\right\} .
\end{aligned}
$$

Using $\tilde{W}(k)=\hat{W}(k)-W^{*}$, we have

$$
2 \tilde{W}^{T}(k) \hat{W}(k)=\tilde{W}^{T}(k) \tilde{W}(k)+\left\|\hat{W}^{T}(k)\right\|^{2}-\left\|W^{*}\right\|^{2} .
$$

It follows that the first difference of $\Delta V_{\tilde{W}}(k)$ along the above equation is

$$
\begin{aligned}
\Delta V_{\tilde{W}}(k)=\mathbb{E} & \left\{\frac{2 \gamma \tilde{W}^{T}(k) S\left(\bar{x}_{n}(k)\right) z_{1}(k)}{1+\left\|S\left(\bar{x}_{n}(k)\right)\right\|^{2} z_{1}^{2}(k)}-\sigma \tilde{W}^{T}(k) \tilde{W}(k)\right. \\
& +\frac{\gamma^{2}\left\|S\left(\bar{x}_{n}(k)\right)\right\|^{2} z_{1}^{2}(k)}{\left[1+\left\|S\left(\bar{x}_{n}(k)\right)\right\|^{2} z_{1}^{2}(k)\right]^{2}}-\sigma(1-\sigma)\|\hat{W}(k)\|^{2} \\
& \left.-\frac{2 \gamma \sigma \hat{W}^{T}(k) S\left(\bar{x}_{n}(k)\right) z_{1}(k)}{1+\left\|S\left(\bar{x}_{n}(k)\right)\right\|^{2} z_{1}^{2}(k)}+\sigma\left\|W^{*}\right\|^{2}\right\} \cdot
\end{aligned}
$$

By observing

$$
\frac{\left\|S\left(\bar{x}_{n}(k)\right)\right\|^{2} z_{1}^{2}(k)}{1+\left\|S\left(\bar{x}_{n}(k)\right)\right\|^{2} z_{1}^{2}(k)} \leq 1
$$

and using the following inequalities

$$
\begin{gathered}
\frac{2 \gamma \tilde{W}^{T}(k) S\left(\bar{x}_{n}(k)\right) z_{1}(k)}{1+\left\|S\left(\bar{x}_{n}(k)\right)\right\|^{2} z_{1}^{2}(k)} \leq \frac{\tilde{W}^{T}(k) \tilde{W}(k)}{4 \varrho}+\varrho \gamma^{2} \\
\frac{\gamma^{2}\left\|S\left(\bar{x}_{n}(k)\right)\right\|^{2} z_{1}^{2}(k)}{\left[1+\left\|S\left(\bar{x}_{n}(k)\right)\right\|^{2} z_{1}^{2}(k)\right]^{2}} \leq \frac{\gamma^{2}}{4} \\
-\frac{2 \gamma \sigma \hat{W}^{T}(k) S\left(\bar{x}_{n}(k)\right) z_{1}(k)}{1+\left\|S\left(\bar{x}_{n}(k)\right)\right\|^{2} z_{1}^{2}(k)} \leq \sigma^{2}\|\hat{W}(k)\|^{2}+\frac{\gamma^{2}}{4}
\end{gathered}
$$

we have

$$
\begin{aligned}
\Delta V_{\tilde{W}}(k) \leq & -\left(\sigma-\frac{1}{4 \varrho}\right) \tilde{W}^{T}(k) \tilde{W}(k)+\sigma\left\|W^{*}\right\|^{2} \\
& -\sigma(1-2 \sigma)\|\hat{W}(k)\|^{2}+(0.5+\varrho) \gamma^{2}
\end{aligned}
$$

By selecting the design parameters to satisfy the following conditions

$$
\frac{1}{4 \varrho}<\sigma<0.5, \quad 0<\gamma<1, \varrho>1
$$

one has

$$
\Delta V_{\tilde{W}}(k) \leq-\beta \tilde{W}^{T}(k) \tilde{W}(k)+\rho_{w}
$$

where $\rho_{w}=\sigma\left\|W^{*}\right\|^{2}+(0.5+\varrho) \gamma^{2}, \beta=\sigma-\frac{1}{4 \varrho}$. It follows easily from (57) that $0<\beta<1$. According to Lemma 1 , the weight estimate error $\tilde{W}(k)$ is EMS bounded and satisfies

$$
\mathbb{E}\left\{\|\tilde{W}(k)\|^{2}\right\} \leq \frac{\lambda_{2}}{\lambda_{1}}\|\tilde{W}(0)\|^{2}(1-\beta)^{k}+\frac{\rho_{w}}{\lambda_{1} \psi}:=\Phi_{w}
$$

where $\tilde{W}(0)$ is the given initial weight vector, $0<\lambda_{1}<1$, $\lambda_{2}>1$. 
Now, we are in the position to state the main result, which shows the uniform boundedness in probability of all closedloop signals by using the proposed adaptive neural controller (48) with weight update law (50).

Theorem 3: Consider the closed-loop system consisting of the discrete-time strict-feedback nonlinear system (1) under Assumptions 1 and 3, the actual controller (48), and the weight update law (50) with design parameters satisfying (57). For any given initial condition, the closed-loop system in the presence of multiplicative noise is EMS bounded if there exist positive constants $p_{i}>0,0<\psi<\beta<1$ and $\mu>1$ such that the following conditions hold:

$p_{i}-p_{i-1} \bar{g}_{i-1}^{2}-2 p_{n} L_{i}-p_{i} \psi>0, \mu \beta-4 p_{n} \bar{g}_{n}^{2} s^{2}-\mu \psi>0$

where $i=1,2, \cdots, n, p_{0}=0, L_{i}>0$ and $\beta$ are respectively defined in (41) and (58).

Proof: Construct the Lyapunov function as follows:

$$
V_{z w}(k)=V_{z}(k)+\mu V_{\tilde{W}}(k)
$$

where $V_{z}(k)=\sum_{i=1}^{n} p_{i} z_{i}^{2}(k), V_{\tilde{W}}(k)$ is given in (52), and $p_{i}$ and $\mu$ are constant coefficients. The difference of $V_{z}(k)$ along (10), (23) and (49) is given by

$$
\begin{aligned}
& \Delta V_{z}(k)=\mathbb{E}\left\{V_{z}(k+1) \mid z(k)\right\}-V_{z}(k) \\
& \leq 4 p_{n} \bar{g}_{n}^{2} \tilde{W}^{T}(k) \tilde{W}(k)\left\|S\left(\bar{x}_{n}(k)\right)\right\|^{2}+4 p_{n} \bar{g}_{n}^{2} \varepsilon^{2} \\
& \quad+2 p_{n} h^{2}\left(\bar{x}_{n}(k)\right)-\sum_{i=1}^{n}\left(p_{i}-p_{i-1} \bar{g}_{i-1}^{2}\right) z_{i}^{2}(k) .
\end{aligned}
$$

Along the similar line for (40)-(41) and noticing $\left\|S\left(\bar{x}_{n}(k)\right)\right\| \leq s$ ( $s$ is a bounded value) given in [21], we have

$$
\begin{aligned}
\Delta V_{z}(k) \leq & 4 p_{n} \bar{g}_{n}^{2} s^{2} \tilde{W}^{T}(k) \tilde{W}(k)+4 p_{n} \bar{g}_{n}^{2} \varepsilon^{2} \\
& +2 p_{n} \sum_{i=1}^{n} L_{i} z_{i}^{2}(k)-\sum_{i=1}^{n}\left(p_{i}-p_{i-1} \bar{g}_{i-1}^{2}\right) z_{i}^{2}(k) \\
= & -\sum_{i=1}^{n}\left(p_{i}-p_{i-1} \bar{g}_{i}^{2}-2 p_{n} L_{i}\right) z_{i}^{2}(k) \\
& +4 p_{n} \bar{g}_{n}^{2} s^{2} \tilde{W}^{T}(k) \tilde{W}(k)+4 p_{n} \bar{g}_{n}^{2} \varepsilon^{2} .
\end{aligned}
$$

By combining (58) and (62), the first difference of $V_{z w}(k)$ in $(60)$ is derived as follows:

$$
\begin{aligned}
\Delta V_{z w}(k) & =\mathbb{E}\left\{\Delta V_{z}(k)\right\}+\mathbb{E}\left\{\Delta V_{\tilde{W}}(k)\right\} \\
\leq & -\sum_{i=1}^{n}\left(p_{i}-p_{i-1} \bar{g}_{i-1}^{2}-2 p_{n} L_{i}\right) z_{i}^{2}(k)+\mu \rho_{w} \\
& -\left(\mu \beta-4 p_{n} s^{2} \bar{g}_{n}^{2}\right) \tilde{W}^{T}(k) \tilde{W}(k)+4 p_{n} \bar{g}_{n}^{2} \varepsilon^{2} .
\end{aligned}
$$

If the constant coefficients are appropriately chosen such that the conditions (59) hold, then we have

$$
\Delta V_{z w}(k) \leq-\psi V_{z w}(k)+\rho_{x w}
$$

where $\rho_{x w}=\mu \rho_{w}+4 p_{n} \bar{g}_{n}^{2} \varepsilon^{2}$. According to Lemma 1, the closed-loop signals $z_{i}(k)$ and $\tilde{W}(k)$ are EMS bounded. Since $\tilde{W}(k)=\hat{W}(k)-W^{*}$, it is clear that $\hat{W}(k)$ is also uniformly ultimately bounded in probability. Since $z_{1}(k)=x_{1}(k)$, we know that $x_{1}(k)$ is bounded in probability. From the coordinate transformations (7), we have $z_{2}(k)=x_{2}(k)-\alpha_{1}(k)$. Noting that $\alpha_{1}(k)=-f_{1}\left(x_{1}(k)\right) / g_{1}\left(x_{1}(k)\right)$ with $f_{1}\left(x_{1}(k)\right)$ and $g_{1}\left(x_{1}(k)\right)$ being smooth functions, $\alpha_{1}(k)$ is bounded and, furthermore, we know that $x_{2}(k)$ is also bounded. Using the similar analysis, it can be concluded that $x_{i}(k)(3 \leq i \leq n)$ and $u(k)$ are bounded in probability. Therefore, all the signals in the closed-loop system are EMS bounded.

Remark 4: In this paper, we develop a new backsteppingbased control framework for a class of discrete-time SFNSs (1) with the multiplicative noise. Such a framework is fundamentally different from the traditional ones using predictor methods proposed in [11], [13], [24]. By using the variable substitution technology and building the relationship between the system states $x_{i}(k)$ and the controlled errors $z_{i}(k)$, the proposed framework overcomes the difficulty in the stability analysis caused by the multiplicative noises, avoids time delays in the neural weight updating law, and reduces the computational burden by employing one RBF neural approximator.

\section{Simulation Results}

In this section, two examples are given to show the validity and applicability of the proposed schemes, respectively, on a second-order SFNS and a direct-current motor.

\section{A. Numerical Example}

To illustrate the effectiveness of the proposed schemes, we first consider a class of discrete-time SFNSs with multiplicative noises as follows:

$$
\left\{\begin{array}{l}
x_{1}(k+1)=g_{1}\left(x_{1}(k)\right) x_{2}(k)+f_{1}\left(x_{1}(k)\right) \\
x_{2}(k+1)=g_{2}\left(\bar{x}_{2}(k)\right) u(k)+f_{2}\left(\bar{x}_{2}(k)\right)+h\left(\bar{x}_{2}(k)\right) \omega(k)
\end{array}\right.
$$

where $x_{1}(k)$ and $x_{2}(k)$ are the system states, $u(k)$ is the system input, $\omega(k)$ is a Gaussian white noise sequence satisfying $\mathbb{E}(\omega(k))=0$ and $\mathbb{E}\left(\omega^{2}(k)\right)=1, f_{i}\left(\bar{x}_{i}(k)\right)$ and $g_{i}\left(\bar{x}_{i}(k)\right), i=1,2$, represent nonlinear dynamics chosen as $f_{1}\left(x_{1}(k)\right)=x_{1}^{2}(k) /\left(1+x_{1}^{2}(k)\right), f_{2}\left(\bar{x}_{2}(k)\right)=x_{2}(k)+$ $\left(0.2 x_{1}(k)-0.6 x_{2}(k)\right) /\left(1+x_{1}^{2}(k)+x_{2}^{2}(k)\right), g_{1}\left(x_{1}(k)\right)=0.5+$ $0.2 \sin \left(x_{1}(k)\right), g_{2}\left(\bar{x}_{2}(k)\right)=1+0.8 \cos \left(x_{1}(k)\right)$, and $h\left(\bar{x}_{2}(k)\right)$ is a randomly occurring nonlinearity. In the simulation, we select $h\left(\bar{x}_{2}(k)\right)=L x_{2}(k) \cos \left(x_{1}(k)\right)$ where $L$ is a Lipschitz constant which is regarded as the intensity of the multiplicative noise $h\left(\bar{x}_{2}(k)\right) \omega(k)$, and $\bar{x}_{2}(k)=\left[x_{1}(k), x_{2}(k)\right]^{T}$. It is easily checked that Assumption 1 is satisfied.

Case 1: Backstepping-Based Control In this case, the functions $f_{i}\left(\bar{x}_{i}(k)\right)$ and $g_{i}\left(\bar{x}_{i}(k)\right), i=1,2$, are exactly known and can be used to construct the ideal controller. For given initial states $x_{1}(0)=0.2$ and $x_{2}(0)=0.5$, the simulation is performed by using the backstepping-based idea controller (29). When $L=1$, the closed-loop state curves are depicted in Fig. 1. Fig. 1 clearly illustrates that the proposed strategy achieves a satisfactory control performance. To evaluate how the intensity $L$ of $h\left(\bar{x}_{2}(k)\right) \omega(k)$ affects the control performance, the simulation is performed by selecting $L=0.3$, $L=1$ and $L=1.5$, respectively. The corresponding results are shown in Figs. 2-4. Fig. 2 and Fig. 3 display the responses of $x_{1}(k)$ and $x_{2}(k)$. The control input signal $u^{*}(k)$ is shown in Fig. 4. From Figs. 2-4, the intensity $L$ of the multiplicative noise $h\left(\bar{x}_{2}(k)\right) \omega(k)$ affects the control performance of the 
closed-loop system to a certain extent. Specifically, the convergence rate of system states becomes faster as the value of $L$ reduces.

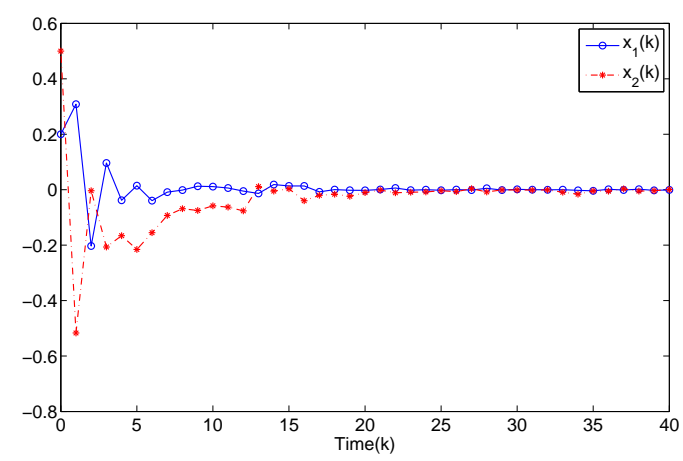

Fig. 1. State curves of the closed-loop system for Section V-A.

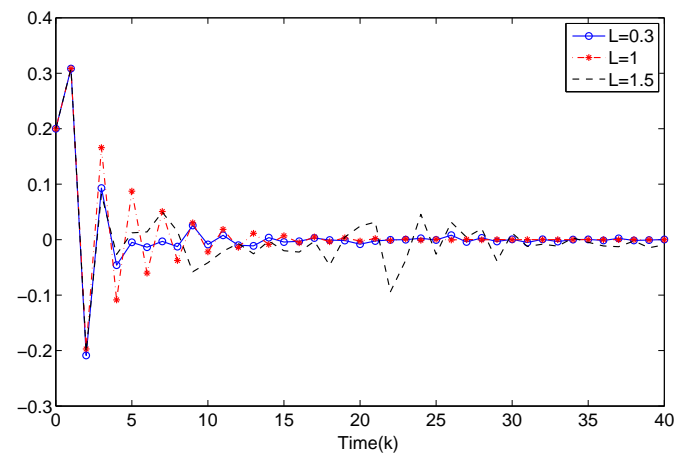

Fig. 2. State curves $x_{1}(k)$ for Case 1 in Section V-A.

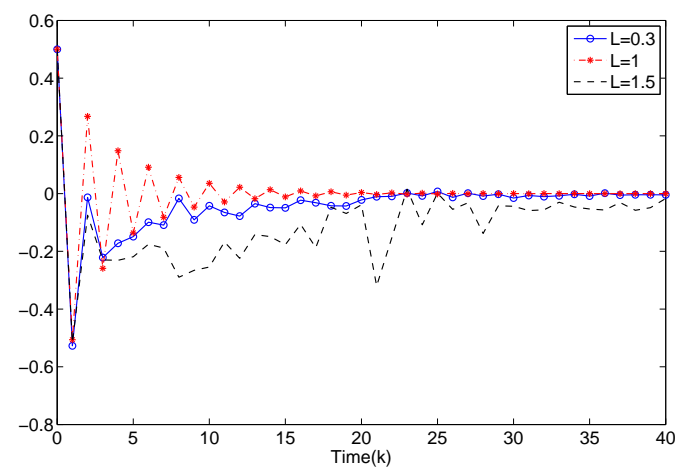

Fig. 3. State curves $x_{2}(k)$ for Case 1 in Section V-A.

Case 2: Adaptive Neural Control In this case, we consider that the system (65) contains the modeling uncertainties $f_{1}\left(x_{1}(k)\right)$ and $f_{2}\left(\bar{x}_{2}(k)\right)$. To handle the modeling uncertainties, the adaptive neural controller (48), (50) is employed to guarantee the closed-loop stability. In the simulation, design parameters are chosen as $\gamma=0.1$ and $\sigma=0.45$, the Gaussian RBF NN $\hat{W}^{T}(k) S\left(\bar{x}_{2}(k)\right)$ is constructed with neural nodes $q=81$, the width 0.12 and the centers evenly spaced on $[-0.1,0.7] \times[-0.1,0.7]$, the initial conditions are set as $x_{1}(0)=0.2$ and $x_{2}(0)=0.5$, and the initial weight vector

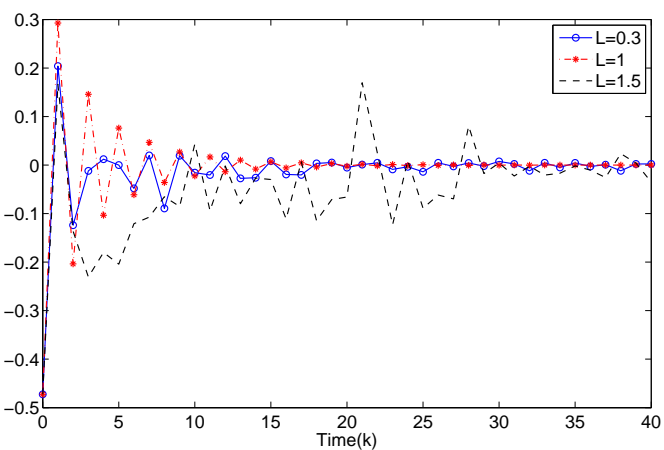

Fig. 4. Control input signal $u^{*}(k)$ for Case 1 in Section V-A.

$\hat{W}(0) \in \mathbb{R}^{81}$ is chosen as a vector with each element being a standard uniform distributed random value divided by 10 . For different given Lipschitz constants $L=0.3, L=1$, and $L=1.5$, simulation results are shown in Figs. 5-7. From Figs. 5-7, all the closed-loop signals are EMS bounded. Moreover, it can be seen from Figs. 5-7 that the smaller intensity of the multiplicative noise $h\left(\bar{x}_{2}(k)\right) \omega(k)$ reaches a better control performance, which is consistent with the stability criteria (36), (59). In terms of computing time, we remove the noise term $h\left(\bar{x}_{2}(k)\right) \omega(k)$ in (69) and compare the control scheme proposed by this paper with the classical $n$-step-ahead predictor method [11]. In the same computing capacity environment, for the same 10000 steps simulation, it takes 1.06 seconds to adopt this paper method and $8.67 \mathrm{sec}-$ onds to adopt the classical $n$-step-ahead predictor method [11]. This comparison shows that the proposed scheme with only one $\mathrm{NN}$ approximator can extremely reduce the computational burden.

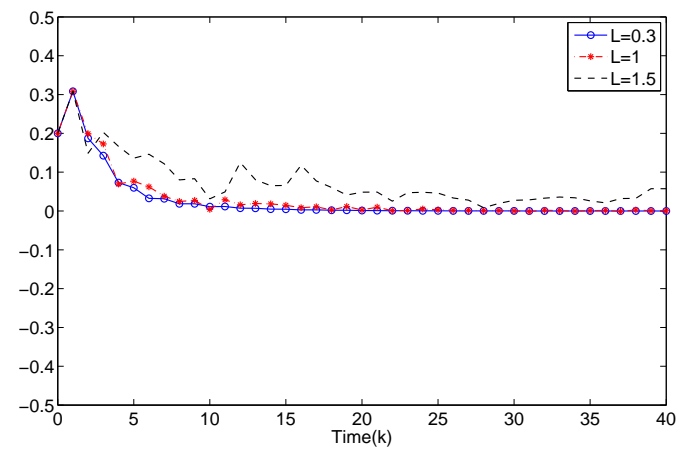

Fig. 5. State curves $x_{1}(k)$ for Case 2 of Section V-A.

\section{B. A DC Motor System}

To demonstrate that the proposed approach can be applied to practical systems, we consider a DC motor system [20] subject to multiplicative noises. The continuous-time dynamical system of the DC motor driven by white noise is described as follows:

$$
\left\{\begin{array}{l}
\mathrm{d} q_{1}=q_{2} \mathrm{~d} t \\
\mathrm{~d} q_{2}=\left[\frac{u-g_{1} q_{2}-g_{2}\left(\bar{q}_{2}\right)}{J}\right] \mathrm{d} t-\frac{h\left(\bar{q}_{2}\right)}{J} \mathrm{~d} w
\end{array}\right.
$$




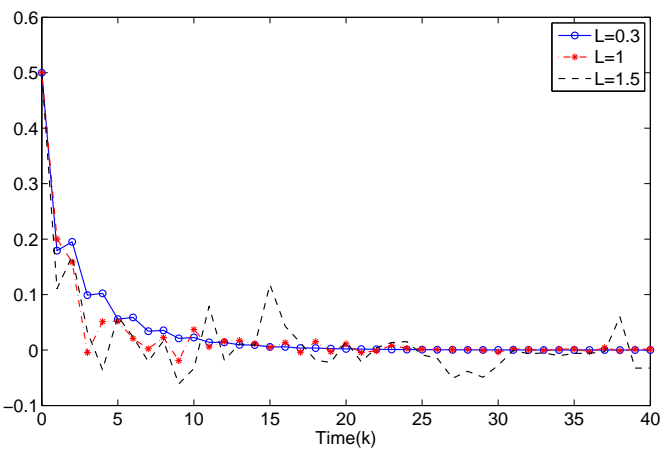

Fig. 6. State curves $x_{2}(k)$ for Case 2 of Section V-A.

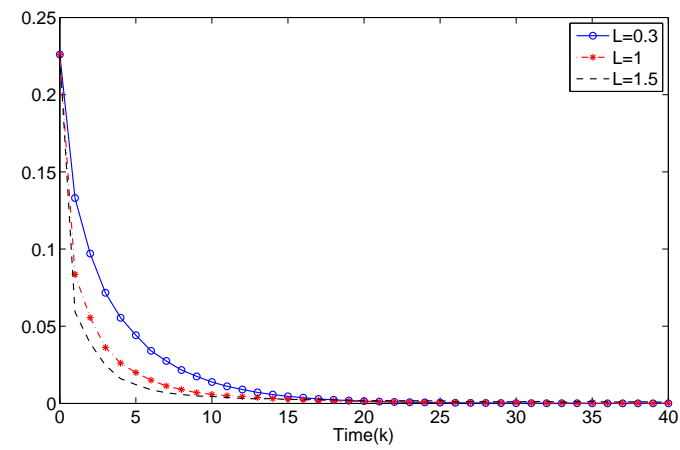

Fig. 7. Control input signal $u(k)$ for Case 2 of Section V-A.

where $q_{1}$ and $q_{2}$ denote respectively the motor angular position and velocity, $u$ is the motor torque, $w$ is a standard Wiener process, $\bar{q}_{2}=\left[q_{1}, q_{2}\right]^{T}, J$ denotes a known moments of inertia, $g_{1}$ is a viscous friction, and $g_{2}\left(\bar{q}_{2}\right)$ and $h\left(\bar{q}_{2}\right)$ denote respectively a nonlinear friction and a randomly occurring nonlinear function, while satisfying $g_{2}(0)=0$ and $h(0)=0$.

Subsequently, by defining $x_{i}=q_{i}, i=1,2$, and using the first-order Taylor expansion, the DC motor system (66) is discretized as follows:

$\left\{\begin{array}{l}x_{1}(k+1)=x_{1}(k)+T x_{2}(k) \\ x_{2}(k+1)=x_{2}(k)+\frac{T}{J}\left[u(k)+f_{2}\left(\bar{x}_{2}(k)\right)-h\left(\bar{x}_{2}(k)\right) w(k)\right]\end{array}\right.$

where the sampling period is chosen as $T=0.1, f_{2}\left(\bar{x}_{2}(k)\right)=$ $-g_{1} x_{2}(k)-g_{2}\left(\bar{x}_{2}(k)\right), \bar{x}_{2}(k)=\left[x_{1}(k), x_{2}(k)\right]^{T}$, and $w(k)$ is

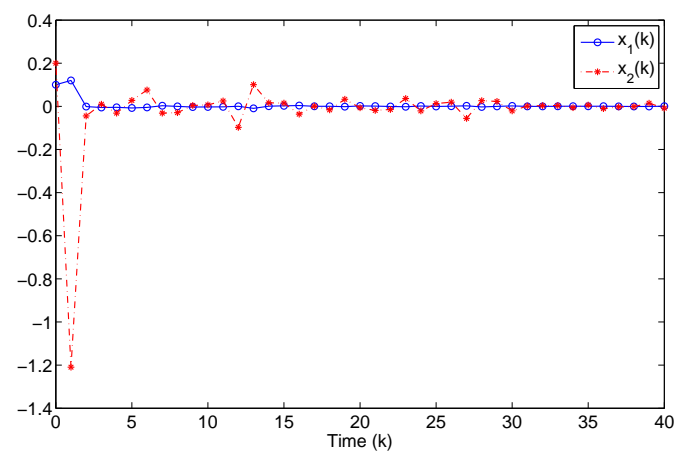

Fig. 8. Motor state curves for the known dynamics.

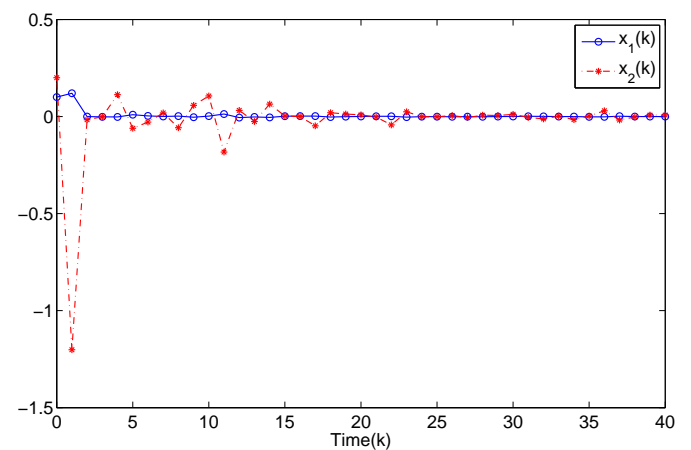

Fig. 9. Motor state curves for the unknown dynamics.

a standard Gaussian white noise sequence. In the simulation, system parameters and nonlinear functions are selected as $J=$ $0.5, g_{1}=0.1, g_{2}\left(\bar{x}_{2}(k)\right)=0.2 x_{1}(k) /\left(1+x_{1}^{2}(k)+x_{2}^{2}(k)\right)$, and $h\left(\bar{x}_{2}(k)\right)=L x_{2}(k) \cos \left(x_{1}(k)\right)$ with $L=4$. From the definition of $f_{2}\left(\bar{x}_{2}(k)\right)$ and $h\left(\bar{x}_{2}(k)\right)$, the DC motor system in a discrete-time form satisfies Assumption 1.

The simulations are performed for both the exact model and the unknown model. Choose the initial states $x_{1}(0)=0.1$ and $x_{2}(0)=0.2$. For the exact model, Fig. 8 shows that the proposed method (29) ensures the mean-square asymptotic convergence of the motor angular position and velocity. For the model with unmodeled dynamics $f_{2}\left(\bar{x}_{2}(k)\right)$, the adaptive neural control scheme (48), (50) is used for the DC motor system (66). In the simulation, we choose design parameters $\gamma=0.1$ and $\sigma=0.45$, construct the Gaussian RBF NN $\hat{W}^{T}(k) S\left(\bar{x}_{2}(k)\right)$ with $\hat{W}(0)=0.01$, neural nodes $q=105$, the neural width 0.15 and the centers evenly spaced on $[-0.1,0.3] \times[-1.5,0.5]$. Fig. 9 illustrates the fact that the proposed adaptive neural control scheme obtains a good control performance even though the considered system (66) contains unmodeled dynamics $f_{2}\left(\bar{x}_{2}(k)\right)$.

Remark 5: It can be seen from (57) that the mean-square bounds of state estimate errors depend on design parameters $\gamma, \sigma$ as well as the node number of RBF NN. In the simulation studies, two principles are taken to achieve good control performance. First of all, the design parameters satisfy $\frac{1}{4 \rho}<\sigma<0.5,0<\gamma<1$. Secondly, the node number of $\mathrm{RBF} N \mathrm{NN}$ is chosen large enough to obtain good approximation performance.

\section{CONCLUSION}

In this paper, a novel backstepping-based control framework has been proposed for a class of discrete-time SFNSs subject to the multiplicative noise. By effectively building the relationship between system states and controlled errors, the proposed framework has simultaneously dealt with the non-causality problem resulting from backstepping design and the difficulty in stability analysis caused by the multiplicative noises. With the help of the proposed framework and exact model information, two kinds of sufficient conditions have been derived to guarantee that the closed-loop system with respect to different multiplicative noises is asymptotically stable in the meansquare sense. When the system under consideration is not exactly modeled, an RBF NN has been employed to approximate the ideal controller, and then a novel adaptive neural 
control scheme has been developed to derive the stability criteria in probability. Such a control scheme not only ensures the mean-square boundedness of the considered systems with modeling uncertainties, but also reduces the computational burden as well as facilitates the implementation using only one neural approximator. The numerical example and DC motor system subject to multiplicative noises have been simulated, respectively, to demonstrate the validity and applicability of the proposed scheme. Furthermore, it is expected that the proposed results can be extended to more general DTSFNSs with different phenomena including prescribed performances [5], [16], constrained network resources [10], [36] and timedelays [37], [45]. The learning mechanism [38], [39] can be also envisaged to be embedded in the framework developed in this paper.

\section{REFERENCES}

[1] C. P. Bechlioulis and G. A. Rovithakis, "Robust adaptive control of feedback linearizable MIMO nonlinear systems with prescribed performance," IEEE Transactions on Automatic Control, vol. 53, no. 9 , pp. 2090-2099, 2008.

[2] C. L. P. Chen, Y.-J. Liu, G.-X. Wen, "Fuzzy neural network-based adaptive control for a class of uncertain nonlinear stochastic systems," IEEE Transactions on Cybernetics, vol. 44, no. 5, pp. 583-593, 2014.

[3] M. Chen, S. S. Ge, and B. Ren, "Adaptive tracking control of uncertain MIMO nonlinear systems with input constraints," Automatica, vol. 47, no. 3, pp. 452-465, 2011.

[4] S.-L. Dai, S. He, H. Lin, and C. Wang, "Platoon formation control with prescribed performance guarantees for USVs," IEEE Transactions on Industrial Electronics, vol. 65, no. 5, pp. 4237-4246, 2018.

[5] S.-L. Dai, M. Wang, and C. Wang, "Neural learning control of marine surface vessels with guaranteed transient tracking performance," IEEE Transactions on Industrial Electronics, vol. 63, no. 3, pp. 1717-1727, 2016.

[6] S. N. Dashkovskiy, and S. S. Pavlichkov, "Global uniform input-tostate stabilization of large-scale interconnections of MIMO generalized triangular form switched systems," Mathematics of Control, Signals, and Systems, vol. 24, no. 1, pp. 135-168, 2012.

[7] S. N. Dashkovskiy, and S. S. Pavlichkov, "Robust stabilization of the generalized triangular form nonlinear systems with disturbances," IEEE Transactions on Automatic Control, vol. 59, no. 6, pp. 1577-1582, 2014.

[8] H. Deng and M. Krstic, "Stochastic nonlinear stabilization-I: backstepping design," Systems \& Control Letters, vol. 32, no. 3, pp. 143-150, 1997.

[9] D. Ding, Z. Wang, J. Lam, and B. Shen, "Finite-horizon H-infinity control for discrete time-varying systems with randomly occurring nonlinearities and fading measurements," IEEE Transactions on Automatic Control, vol. 60, no. 9, pp. 2488-2493, 2015.

[10] H. Dong, Z. Wang, S. X. Ding, and H. Gao, "Event-based $H_{\infty}$ filter design for a class of nonlinear time-varying systems with fading channels and multiplicative noises," IEEE Transactions on Signal Processing, vol. 63, no. 13, pp. 3387-3395, 2015

[11] S. S. Ge, G. Y. Li, and T. H. Lee, "Adaptive NN control for a class of strict-feedback discrete-time nonlinear systems," Automatica, vol. 39, no. 5, pp. 807-819, 2003.

[12] S. S. Ge, F. Hong, and T. H. Lee, "Adaptive neural network control of nonlinear systems with unknown time delays," IEEE Transactions on Automatic Control, vol. 48, no. 11, pp. 2004-2010, 2003.

[13] S. S. Ge, C. Yang, and T. H. Lee, "Adaptive predictive control using neural network for a class of pure-feedback systems in discrete time," IEEE Transactions on Neural Networks, vol. 19, no. 9, pp. 1599-1614, 2008.

[14] J. Hu, Z. Wang, S. Liu, H. Gao, "A variance-constrained approach to recursive state estimation for time-varying complex networks with missing measurements," Automatica, vol. 64, pp. 155-162, 2016

[15] W. He, A. O. David, Z. Yin, and C. Sun, "Neural network control of a robotic manipulator with input deadzone and output constraint," IEEE Transactions on Systems, Man, and Cybernetics: Systems, vol. 46, no. 6, pp. 759-770, 2016.

[16] S. He, M. Wang, S. Dai, and F. Luo, "Leader-follower formation control of USVs with prescribed performance and collision avoidance," IEEE Transactions on Industrial Informatics, vol. 15, no. 1, pp. 572-581, 2019.

[17] Z.-P. Jiang and D. J. Hill, "A robust adaptive backstepping scheme for nonlinear systems with unmodeled dynamics," IEEE Transactions on Automatic Control, vol. 44, no. 9, pp. 1705-1711, 1999
[18] H. K. Khalil, Nonlinear Systems, 3rd ed. Upper Saddle River, NJ: Prentice hall, 2002

[19] M. Krstic, I. Kanellakopoulos, and P. V. Kokotovic, Nonlinear and Adaptive Control Design. New York: Wiley, 1995.

[20] R. Krishnan, Electronic Motor Drives: Modeling, Analysis and Control. Upper Saddle River, NJ: Prentice hall, 2001.

[21] A. J. Kurdila, F. J. Narcowich, and J. D. Ward, "Persistency of excitation in identification using radial basis function approximants," SIAM Journal on Control and Optimization, vol. 32, no. 2, pp. 625-642, 1995.

[22] F. L. Lewis, A. Yesildirek, and K. Liu, "Robust backstepping control of induction motors using neural networks," IEEE Transactions on Neural Networks, vol. 11, no. 5, pp. 1178-1187, 2000.

[23] X. Liu, A. Jutan, and S. Rohani, "Almost disturbance decoupling of MIMO nonlinear systems and application to chemical processes," Automatica, vol. 40, no. 3, pp. 465-471, 2004.

[24] Y.-J. Liu, T. Tang, S. Tong, and C. L. P. Chen, "Adaptive NN controller design for a class of nonlinear MIMO discrete-time systems," IEEE Transactions on Neural Networks and Learning Systems, vol. 26, no. 5, pp. 1007-1018, 2015.

[25] L. Ma, Z. Wang, Q.-L. Han, and H.-K. Lam, "Variance-constrained distributed filtering for time-varying systems with multiplicative noises and deception attacks over sensor networks," IEEE Sensors Journal, vol. 17, no. 7, pp. 2279-2288, 2017.

[26] D. Nodland, H. Zargarzadeh, and S. Jagannathan, "Neural network-based optimal adaptive output feedback control of a helicopter UAV," IEEE Transactions on Neural Networks and Learning Systems, vol. 24, no. 7, pp. 1061-1073, 2013.

[27] Z. Pan and T. Basar, "Adaptive controller design for tracking and disturbance attenuation in parametric strictfeedback nonlinear systems," IEEE Transactions on Automatic Control, vol. 43, no. 8, pp. 1066-1083, 1998.

[28] S. S. Pavlichkov, and S. S. Ge, "Global stabilization of the generalized MIMO triangular systems with singular input-output links," IEEE Transactions on Automatic Control, vol. 54, no. 8, pp. 1794-1806, 2009.

[29] M. Saif, B. Liu, and H. Fan, "Stabilisation and control of a class of discrete-time nonlinear stochastic output-dependent system with random missing measurements," International Journal of Control, vol. 90, no. 8, pp. 1678-1687, 2017.

[30] R. M. Sanner and J. E. Slotine, "Gaussian networks for direct adaptive control," IEEE Transactions on Neural Networks, vol. 3, no. 6, pp. 837 863, 1992.

[31] Y. Su and J. Huang, "Cooperative global robust output regulation for nonlinear uncertain multi-agent systems in lower triangular form," IEEE Transactions on Automatic Control, vol. 60, no. 9, pp. 2378-2389, Sept. 2015.

[32] K. P. Tee, S. S. Ge, and E. H. Tay, "Barrier Lyapunov functions for the control of output-constrained nonlinear systems," Automatica, vol. 45, no. 4, pp. 918-927, 2009.

[33] S. Tong and Y. Li, "Robust adaptive fuzzy backstepping output feedback tracking control for nonlinear system with dynamic uncertainties," Science China Information Sciences, vol. 53, no. 2, pp. 307-324, 2010

[34] J. Vance and S. Jagannathan, "Discrete-time neural network output feedback control of nonlinear discrete-time systems in non-strict form,' Automatica, vol. 41, no. 6, pp. 1020-1027, 2008.

[35] H. Wang, B. Chen, X. Liu, K. Liu, and C. Lin, "Adaptive neural tracking control for stochastic nonlinear strict-feedback systems with unknown input saturation," Information Sciences, vol. 269, no. 10, pp. 300-315, 2014.

[36] L. Wang, Z. Wang, Q.-L. Han, and G. Wei, "Event-based varianceconstrained $H_{\infty}$ filtering for stochastic parameter systems over sensor networks with successive missing measurements," IEEE Transactions on Cybernetics, vol. 48, no. 3, pp. 1007-1017, 2018.

[37] M. Wang, S. S. Ge, and K.-S. Hong, "Approximation-based adaptive tracking control of pure-feedback nonlinear systems with multiple unknown time-varying delays," IEEE Transactions on Neural Networks, vol. 21, no. 11, pp. 1804-1816, 2010.

[38] M. Wang, C. Wang, P. Shi, and X. Liu, "Dynamic learning from neural control for strict-feedback systems with guaranteed predefined performance," IEEE Transactions on Neural Networks and Learning Systems, vol. 27, no. 12, pp. 2564-2576, 2016.

[39] M. Wang and A. Yang, "Dynamic learning from adaptive neural control of robot manipulators with prescribed performance," IEEE Transactions on Systems, Man, and Cybernetics: Systems, vol. 47, no. 8, pp. 2244 2255,2017

[40] Z.-J. Wu, X.-J. Xie, and S.-Y. Zhang, "Adaptive backstepping controller design using stochastic small-gain theorem," Automatica, vol. 43, no. 4, pp. 608-620, 2007.

[41] B. Xu, C. Yang, and Z. Shi, "Reinforcement Learning Output Feedback NN Control Using Deterministic Learning Technique," IEEE Transactions on Neural Networks and Learning Systems, vol. 25, no. 3, pp. 635$641,2014$. 
[42] C. Yang, S. S. Ge, C. Xiang, T. Chai, and T. H. Lee, "Output feedback NN control for two classes of discrete-time systems with unknown control directions in a unified approach," IEEE Transactions on Neural Networks, vol. 19, no. 11, pp. 1873-1886, 2008.

[43] F. Yang, Z. Wang, Y. S. Hung, and M. Gani, " $H_{\infty}$ control for networked systems with random communication delays," IEEE Transactions on Automatic Control, vol. 51, no. 3, pp. 511-518, 2006.

[44] P. C. Yeh and P. V. Kokotovic, "Adaptive control of a class of nonlinear discrete-time systems," International Journal of Control, vol. 2, no. 62, pp. 303-324, 1995.

[45] S. J. Yoo, J. B. Park, and Y. H. Choi, "Adaptive neural control for a class of strict-feedback nonlinear systems with state time delays," IEEE Transactions on Neural Networks, vol. 20, no. 7, pp. 1209-1215, 2009.

[46] Y. Yuan, Z. Wang, P. Zhang, and H. Dong, "Nonfragile near-optimal control of stochastic time-varying multiagent systems with control- and state-dependent noises," IEEE Transactions on Cybernetics, vol. 49, no. 7, pp. 2605-2617, 2019.

[47] Y. Zhang, C. Wen, and Y. Soh, "Discrete-time robust backstepping adaptive control for nonlinear time-varying systems," IEEE Transactions on Automatic Control, vol. 45, no. 9, pp. 1749-1755, 2000.

[48] Q. Zhou, S. Zhao, H. Li, R. Lu and C. Wu, "Adaptive neural network tracking control for robotic manipulators with dead-zone," IEEE Transactions on Neural Networks and Learning Systems, vol. 30, no. 12, pp. 3611-3620, 2019 .

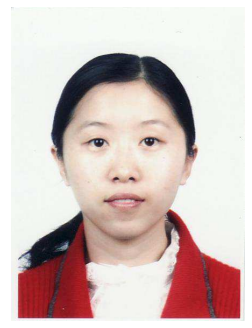

Min Wang (M'09) received the B.Sc. degree in mathematics and the M.Sc. degree in applied mathematics from Bohai University, Jinzhou, China, in 2003 and 2006, respectively, and the Ph.D. degree in system theory from Qingdao University, Qingdao, China, in 2009

She was a Visiting Scholar with the Department of Computer Science, Brunel University London, Uxbridge, U.K., from 2017 to 2018 . She is currently a Professor with the School of Automation Science and Engineering, South China University of Technology, Guangzhou, China. She has authored or coauthored over 40 papers published in international journals. Her current research interests include intelligent control, dynamic learning, robot control, and event-triggered control.

Dr. Wang is currently an Associate Editor of International Journal of Systems Science, IEEE Access, and Control Theory and Applications.

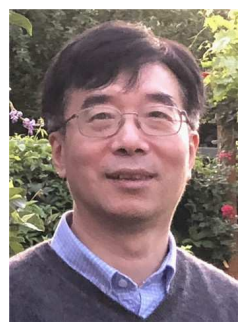

Zidong Wang (SM'03-F'14) was born in Jiangsu, China, in 1966. He received the B.Sc. degree in mathematics in 1986 from Suzhou University, Suzhou, China, and the M.Sc. degree in applied mathematics in 1990 and the Ph.D. degree in electrical engineering in 1994, both from Nanjing University of Science and Technology, Nanjing, China.

$\mathrm{He}$ is currently Professor of Dynamical Systems and Computing in the Department of Computer Science, Brunel University London, U.K. From 1990 to 2002 , he held teaching and research appointments in universities in China, Germany and the UK. Prof. Wang's research interests include dynamical systems, signal processing, bioinformatics, control theory and applications. He has published around 220 papers in IEEE Transactions and around 60 papers in Automatica. He is a holder of the Alexander von Humboldt Research Fellowship of Germany, the JSPS Research Fellowship of Japan, William Mong Visiting Research Fellowship of Hong Kong.

Prof. Wang serves (or has served) as the Editor-in-Chief for Neurocomputing, the Deputy Editor-in-Chief for International Journal of Systems Science, and an Associate Editor for 12 international journals including IEEE Transactions on Automatic Control, IEEE Transactions on Control Systems Technology, IEEE Transactions on Neural Networks, IEEE Transactions on Signal Processing, and IEEE Transactions on Systems, Man, and CyberneticsPart C. He is a Fellow of the IEEE, a Fellow of the Royal Statistical Society and a member of program committee for many international conferences.

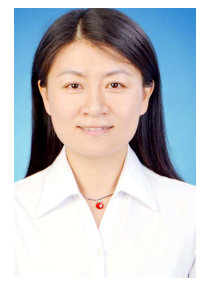

Hongli Dong (SM'16) received the Ph.D. degree in control science and engineering from the Harbin Institute of Technology, Harbin, China, in 2012

From 2009 to 2010, she was a Research Assistant with the Department of Applied Mathematics, City University of Hong Kong, Hong Kong. From 2010 to 2011, she was a Research Assistant with the Department of Mechanical Engineering, The University of Hong Kong, Hong Kong. From 2011 to 2012, she was a Visiting Scholar with the Department of Information Systems and Computing, Brunel University London, London, U.K. From 2012 to 2014, she was an Alexander von Humboldt Research Fellow with the University of Duisburg-Essen, Duisburg, Germany. She is currently a Professor with the Institute of Complex Systems and Advanced Control, Northeast Petroleum University, Daqing, China. She is also the Director of the Heilongjiang Provincial Key Laboratory of Networking and Intelligent Control, Daqing. Her current research interests include robust control and networked control systems.

Dr. Dong is a very active reviewer for many international journals.

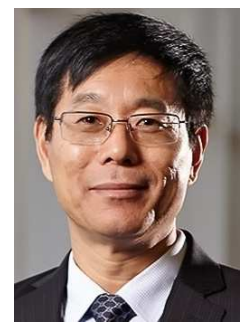

Qing-Long Han (M'09-SM'13-F'19) received the B.Sc. degree in Mathematics from Shandong Normal University, Jinan, China, in 1983, and the M.Sc. and $\mathrm{Ph} . \mathrm{D}$. degrees in Control Engineering and Electrical Engineering from East China University of Science and Technology, Shanghai, China, in 1992 and 1997, respectively.

From 1997 to 1998, he was a Post-doctoral Researcher Fellow with the Laboratoire d'Automatique et d'Informatique Industielle (currently, Laboratoire d'Informatique et d'Automatique pour les Systémes), École Supérieure d'Ing'enieurs de Poitiers (currently, École Nationale Supérieure d'Ingénieurs de Poitiers), Université de Poitiers, France. From 1999 to 2001, he was a Research Assistant Professor with the Department of Mechanical and Industrial Engineering, Southern Illinois University at Edwardsville, Edwardsville, IL, USA. From 2001 to 2014, he was a Laureate Professor, an Associate Dean of research and innovation with the Higher Education Division, and the Founding Director of the Centre for Intelligent and Networked Systems, Central Queensland University, Rockhampton, QLD, Australia. From 2014 to 2016, he was a Deputy Dean of research with the Griffith Sciences and a Professor with the Griffith School of Engineering, Griffith University, Mount Gravatt, QLD, Australia. In 2016, he joined the Swinburne University of Technology, Melbourne, VIC, Australia, where he is currently a Pro Vice-Chancellor of research quality and a Distinguished Professor. His research interests include networked control systems, multiagent systems, time-delay systems, complex dynamical systems, and neural networks.

Professor Han is a Highly Cited Researcher according to Clarivate Analytics (formerly, Thomson Reuters). He is an Associate Editor of several international journals, including the IEEE TRANSACTIONS ON CYBERNETICS, the IEEE TRANSACTIONS ON INDUSTRIAL ELECTRONICS, the IEEE TRANSACTIONS ON INDUSTRIAL INFORMATICS, IEEE Industrial Electronics Magazine, the IEEE/CAA JOURNAL OF AUTOMATICA SINICA, Control Engineering Practice, and Information Sciences. He is a fellow of the Institution of Engineers Australia. 